\title{
Model-Based Safe Reinforcement Learning with Time-Varying State and Control Constraints: An Application to Intelligent Vehicles
}

This paper was downloaded from TechRxiv (https://www.techrxiv.org).

\section{LICENSE}

CC BY 4.0

SUBMISSION DATE / POSTED DATE

$15-12-2021 / 21-12-2021$

\section{CITATION}

Zhang, Xinglong; Peng, Yaoqian; Luo, Biao; Pan, Wei; Xu, Xin; Xie, Haibin (2021): Model-Based Safe Reinforcement Learning with Time-Varying State and Control Constraints: An Application to Intelligent Vehicles. TechRxiv. Preprint. https://doi.org/10.36227/techrxiv.17205740.v2

$\mathrm{DOI}$ 


\title{
Model-Based Safe Reinforcement Learning with Time-Varying State and Control Constraints: An Application to Intelligent Vehicles
}

\author{
Xinglong Zhang Member, IEEE, Yaoqian Peng, Biao Luo Senior member, IEEE, Wei Pan Member, IEEE, Xin \\ $\mathrm{Xu}$ Senior member, IEEE, and Haibin Xie
}

\begin{abstract}
Recently, barrier function-based safe reinforcement learning (RL) with the actor-critic structure for continuous control tasks has received increasing attention. It is still challenging to learn a near-optimal control policy with safety and convergence guarantees. Also, few works have addressed the safe RL algorithm design under time-varying safety constraints. This paper proposes a model-based safe RL algorithm for optimal control of nonlinear systems with time-varying state and control constraints. In the proposed approach, we construct a novel barrier-based control policy structure that can guarantee control safety. A multi-step policy evaluation mechanism is proposed to predict the policy's safety risk under time-varying safety constraints and guide the policy to update safely. Theoretical results on stability and robustness are proven. Also, the convergence of the actor-critic learning algorithm is analyzed. The performance of the proposed algorithm outperforms several state-of-the-art RL algorithms in the simulated Safety Gym environment. Furthermore, the approach is applied to the integrated path following and collision avoidance problem for two real-world intelligent vehicles. A differential-drive vehicle and an Ackermann-drive one are used to verify the offline deployment performance and the online learning performance, respectively. Our approach shows an impressive sim-to-real transfer capability and a satisfactory online control performance in the experiment.
\end{abstract}

Index Terms-safe reinforcement learning, actor-critic algorithm, barrier functions, multi-step policy evaluation, timevarying constraints.

\section{INTRODUCTION}

Reinforcement learning (RL) is promising for solving nonlinear optimal control problems and has received significant attention in the past decades, see [1], [2] and the references therein. Until recently, significant progress has been made on RL with the actor-critic structure for continuous control tasks [1], [3]-[8]. In actor-critic RL, the value function and control policy are represented by the critic and actor networks, respectively, and learned via extensive policy exploration and exploitation. However, the resulting learning-based control system might not guarantee safety for systems with state and stability constraints. It is known that safety constraint satisfaction is crucial besides optimality in many real-world robot

This work has been submitted to the IEEE for possible publication. Copyright may be transferred without notice, after which this version may no longer be accessible. Xinglong Zhang, Yaoqian Peng, Xin Xu, and Haibin Xie are with the College of Intelligence Science and Technology, National University of Defense Technology, Changsha, China. Biao Lu is with the School of Automation, Central South University, Changsha, China. Wei Pan is with the Department of Cognitive Robotics, Delft University of Technology, the Netherlands. Corresponding author: Xin Xu (xinxu@nudt.edu.cn). control applications [9], [10]. For instance, autonomous driving has been viewed as a promising technology that will bring fundamental changes to everyday life. Still, one of the crucial issues concerns how to learn to drive safely under dynamic and unknown environments with unexpected obstacles. For these practical reasons, many safe RL algorithms have been recently developed for safety-critical systems, see e.g. [9]-[26] and the references therein. Note that there are fruitful works in adaptive control with constraints, but the technique used is different from that in RL, for related references in adaptive control might refer to [27], [28].

In general, current safe RL solutions can be categorized into three main approaches. (i) The first family utilizes a unique mechanism in the learning procedure for safe policy optimization using, e.g., control barrier functions [16], [22], [29], formal verification [30], [31], shielding [32]-[34], and external intervention [35], [36]. These methods are prone to a safe-biased learning by sacrificing greatly on the performance. And some of them rely on extra human interference [35], [36]. (ii) The second family proposes safe RL algorithms via primal-dual methods [15], [37]-[39]. In the resulting optimization problem, the Lagrangian multiplier serves as an extra weight whose update is noted sensitive to the control performance [15]. Moreover, some optimization problems such as those with ellipsoidal constraints (covered in this work) could not satisfy the strong duality condition [40]. (iii) The third is reward/cost shaping-based RL approaches [41]-[44] where the cost functions are augmented with various safetyrelated parts, e.g., barrier functions. As stated in [38], such a design only informs the goal of guaranteeing safety by minimizing the reshaped cost function but fails to guide how to achieve it well through an actor-critic structure design. Consequently, weights of actor and critic networks are prone to divergence in the training process due to the abrupt changes of the cost function caused by the safety-related terms in approaching the constraint boundary. These issues motivated our barrier function-based (simplified as barrier-based) actorcritic structure. Moreover, few works have addressed the safe RL algorithm design under time-varying safety constraints.

This work proposes a model-based safe RL algorithm with theoretical guarantees for optimal control with time-varying state and control constraints. A new barrier-based control policy (BCP) structure is constructed in the proposed safe RL approach, generating repulsive control forces as states and controls move toward the constraint boundaries. Moreover, 
the time-varying constraints are addressed by a multi-step policy evaluation (MPE). The closed-loop theoretical property of our approach under nominal and perturbed cases and the convergence condition of the barrier-based actor-critic (BAC) learning algorithm is derived. The effectiveness of our approach is tested on both simulations and real-world intelligent vehicles. Our contributions are summarized as follows.

1) We proposed a safe RL for optimal control under timevarying state and control constraints. Under certain conditions (see Sections IV-A and -B), safety can be guaranteed in both online and offline learning scenarios. The performance and advantages of the proposed approach are achieved by two novel designs. The first is a barrierbased control policy to ensure safety with an actor-critic structure. The second is a multi-step evaluation mechanism to predict the control policy's future influence on the value function under time-varying safety constraints and guide the policy to update safely.

2) We proved that the proposed safe RL could guarantee stability and robustness in the nominal scenario and under external disturbances, respectively. And the convergence condition of the BAC learning algorithm was derived by the Lyapunov method.

3) The proposed approach was applied to solve an integrated path following and collision avoidance problem of intelligent vehicles so that the control performance can be optimized with theoretical guarantees even with external disturbances. (i) Extensive simulation results illustrate that our approach outperforms other state-ofthe-art safe RL methods in learning safety and performance. (ii) We verified our control policy's offline simto-real transfer capability and real-world online learning performance. The experimental results reveal that our approach outperforms a standard model predictive control (MPC) algorithm in terms of safety and optimality and shows an impressive sim-to-real transfer capability and a satisfactory online control performance.

The remainder of the paper is organized as follows. Section II introduces the considered control problem and preliminary solutions. Section III presents the proposed safe RL approach and the BAC learning algorithm, while Section IV presents the main theoretical results. Section V shows the real-world experimental results, while some conclusions are drawn in Section VI. Some additional simulation results for theoretical verification are given in the appendix.

Notation: We denote $\mathbb{N}$ and $\mathbb{N}_{a}^{b}$ as the set of natural numbers and integers $a, a+1, \cdots, b$. For a vector $x \in \mathbb{R}^{n}$, we denote $\|x\|_{Q}^{2}$ as $x^{\top} Q x$ and $\|x\|$ as the Euclidean norm. For a function $f(x)$ with an argument $x$, we denote $\nabla f(x)$ as the gradient to $x$. For a function $f(x, u)$ with arguments $x$ and $u$, we denote $\nabla_{z} f(x, u)$ as the partial gradient to $z, z=x$ or $u$. Given a matrix $A \in \mathbb{R}^{n \times n}$, we use $\lambda_{\min }(A)\left(\lambda_{\max }(A)\right)$ to denote the minimal (maximal) eigenvalues. We denote $\operatorname{Int}(\mathcal{Z})$ as the interior of a general set $\mathcal{Z}$. For variables $z_{i} \in \mathbb{R}^{q_{i}}, i \in \mathbb{N}_{1}^{M}$, we define $\left(z_{1}, z_{2}, \cdots, z_{\mathrm{M}}\right)=\left[\begin{array}{llll}z_{1}^{\top} & z_{2}^{\top} & \cdots & z_{\mathrm{M}}^{\top}\end{array}\right]^{\top} \in \mathbb{R}^{q}$, where $q=\sum_{i=1}^{M} q_{i}$.

\section{CONTROL PROBLEM FORMULATION AND DEFINITIONS}

\section{A. Control problem}

The considered system under control is a class of discretetime nonlinear systems described by

$$
x_{k+1}=f\left(x_{k}, u_{k}\right)
$$

where $x_{k} \in \mathcal{X}_{k} \subseteq \mathbb{R}^{n}$ and $u_{k} \in \mathcal{U}_{k} \subseteq \mathbb{R}^{m}$ are the state and input variables, $k$ is the discrete-time index, $\mathcal{X}_{k}=\{x \in$ $\left.\mathbb{R}^{n} \mid G_{x, k}^{i}(x) \leq 0, \forall i \in \mathbb{N}_{1}^{p_{x}}\right\}$ and $\mathcal{U}_{k}=\left\{u \in \mathbb{R}^{m} \mid G_{u, k}^{i}(u) \leq\right.$ $\left.0, \forall i \in \mathbb{N}_{1}^{p_{u}}\right\}$ are convex sets that represent time-varying constraints, $\{0\} \subseteq \mathcal{U}_{k}, \forall k \in \mathbb{N}, \mathcal{W}$ is a bounded compact set; functions $G_{z, k}^{i}(z) \in \mathbb{R}$ for $z=x, u$, are assumed to be $C^{2} ; f$ is the state transition function and $f(0,0)=0$.

Starting from any initial condition $x_{0} \in \mathcal{X}_{0}$, the control objective is to find an optimal control policy $u_{k}=u\left(x_{k}\right) \in \mathcal{U}_{k}$ that minimizes a quadratic regulation cost function of type

$$
J\left(x_{0}, u_{0:+\infty}\right)=\sum_{k=0}^{+\infty} \gamma^{k} r\left(x_{k}, u_{k}\right)
$$

subject to model (1), $x_{k} \in \mathcal{X}_{k}$, and $u_{k} \in \mathcal{U}_{k}, \forall k \in \mathbb{N}$; where

$$
r\left(x_{k}, u_{k}\right)=\left\|x_{k}\right\|_{Q}^{2}+\left\|u_{k}\right\|_{R}^{2},
$$

and $Q=Q^{\top} \in \mathbb{R}^{n \times n}, R=R^{\top} \in \mathbb{R}^{m \times m}, Q, R \succ 0, \gamma$ is a discount factor.

Definition 1 (Local stabilizability [40]): System (1) with $w=0$ is stabilizable on $\mathcal{X}_{k} \times \mathcal{U}_{k}$ if, for any $x_{0} \in \mathcal{X}_{k}$, there exists a $C^{1}$ state-feedback policy $u\left(x_{k}\right) \in \mathcal{U}_{k}, u(0)=0$, such that $x_{k} \in \mathcal{X}_{k}$ and $x_{k} \rightarrow 0$ as $k \rightarrow+\infty$.

Without loss of generality, many waypoint tracking problems in the robot control field can be naturally formed as the prescribed regulation one, with a proper coordination transformation of the reference waypoints. More generally, it is allowed that the time-varying state constraint might not contain the origin for some $k \in \mathbb{N}$. Typical examples can be found in, for instance, path following of mobile robots with collision avoidance, where the potential obstacle to be avoided might occupy the reference waypoints, i.e., the origin after coordination transformation. It is still reasonable to introduce the following assumption for convergence guarantee.

Assumption 1 (State constraint): There exists a finite number $\bar{k} \in \mathbb{N}$ such that $\{0\} \subseteq \mathcal{X}_{k}$ as $k \geq \bar{k}$.

Assumption 2 (Lipschitz continuous): Model (1) is Lipschitz continuous in $\mathcal{X}_{k} \times \mathcal{U}_{k}$, for all $k \in \mathbb{N}_{1}^{\infty}$, i.e., there exists a Lipschitz constant $0<L_{f}<+\infty$ such that for all $x_{1}, x_{2} \in$ $\mathcal{X}_{k}$ and $C^{1}$ control policies with $u\left(x_{1}\right), u\left(x_{2}\right) \in \mathcal{U}_{k}$,

$$
\left\|f\left(x_{1}, u\left(x_{1}\right)\right)-f\left(x_{2}, u\left(x_{2}\right)\right)\right\| \leq L_{f}\left\|x_{1}-x_{2}\right\| .
$$

Assumption 3 (Model): $\left\|\nabla_{u} f(x, u)\right\| \leq g_{m}$ in the domain $\mathcal{X}_{k} \times \mathcal{U}_{k}$, where $g_{m}>0$.

Definition 2 (multi-step safe control): For a given state $x_{k} \in$ $\mathcal{X}_{k}$ at time instant $k$, a control policy $u\left(x_{k}\right) \in \mathcal{U}_{k}$, is $L$-step safe for (1) if the resulting future state evolutions of (1) under $u\left(x_{k}\right)$ satisfy $x_{k+i} \in \mathcal{X}_{k+i}^{u}, \forall i \in \mathbb{N}_{1}^{L}$, where $\mathcal{X}_{k+i}^{u}$ is the resulting state constraint under $u\left(x_{k}\right)$.

To simplify the notation, in the rest of the paper, the super index in $\mathcal{X}_{k}^{u}$ is neglected, i.e., we use $\mathcal{X}_{k}$ to denote $\mathcal{X}_{k}^{u}$. 


\section{B. Definitions on barrier functions}

We also introduce the definition of barrier functions.

Definition 3 (Barrier function): For a convex set $\mathcal{Z}_{k}=\{z \in$ $\left.\mathbb{R}^{l} \mid G_{z, k}^{i}(z) \leq 0, \forall i \in \mathbb{N}_{1}^{p_{z}}\right\}$, a barrier function is defined as

$$
\mathcal{B}_{k}^{o}(z)=\left\{\begin{array}{l}
-\sum_{i=1}^{p_{z}} \log \left(-G_{z, k}^{i}(z)\right), \quad z \in \operatorname{Int}\left(\mathcal{Z}_{k}\right) \\
+\infty \text { otherwise. }
\end{array}\right.
$$

The recentered transformation of $\mathcal{B}_{k}^{o}(z)$ centered at $z_{c} \in \mathbb{R}^{l}$ is defined as $\mathcal{B}_{k}^{c}(z)=\mathcal{B}_{k}^{o}(z)-\mathcal{B}_{k}^{o}\left(z_{c}\right)-\nabla_{z} \mathcal{B}_{k}^{o}\left(z_{c}\right)^{\top} z$, where $z_{c}=0$ if $\{0\} \subseteq \mathcal{Z}_{k}$ or $z_{c}$ is selected such that $z_{c} \in \mathcal{Z}_{k}$ otherwise.

To derive a satisfactory control performance, it is suggested to select $z_{c}$ far from the set boundary of $\mathcal{Z}_{k}$ and as the central point or its neighbor of $\mathcal{Z}_{k}$ (if possible). It is observed that $\mathcal{B}_{k}^{c}(z)$ satisfies

$$
\mathcal{B}_{k}^{c}\left(z_{c}\right)=0 \text { and } \nabla \mathcal{B}_{k}^{c}\left(z_{c}\right)=0 .
$$

Lemma 1 (Relaxed barrier function [45]):

Define a relaxed barrier function of $\mathcal{B}_{k}^{c}(z)$ as

$$
\mathcal{B}_{k}(z)= \begin{cases}\mathcal{B}_{k}^{c}(z) & \bar{\sigma}_{k} \geq \kappa_{b} \\ \gamma_{b}\left(z, \bar{\sigma}_{k}\right) & \bar{\sigma}_{k}<\kappa_{b}\end{cases}
$$

where $\kappa_{b}>0$ is a relaxing factor, $\bar{\sigma}_{k}=\min _{i \in \mathbb{N}_{1}^{p z}}-G_{k}^{i}(z)$, the function $\gamma_{b}\left(z, \bar{\sigma}_{k}\right)$ is strictly monotone and differentiable on $\left(-\infty, \kappa_{b}\right)$, and $\nabla_{z}^{2} \gamma_{b}\left(z, \bar{\sigma}_{k}\right) \leq\left.\nabla_{z}^{2} \mathcal{B}_{k}(z)\right|_{\bar{\sigma}_{k}=\kappa_{b}}$, then there exists a matrix $H_{z_{k}} \geq\left.\nabla_{z}^{2} \mathcal{B}_{k}(z)\right|_{\bar{\sigma}_{k}=\kappa_{b}}$, such that $\left\|\nabla_{z} \mathcal{B}_{k}(z)\right\| \leq$ $\mathcal{B}_{z_{k}, m}, \mathcal{B}_{z_{k}, m}=\max _{z \in \mathcal{Z}_{k}}\left\|2 H_{z_{k}}\left(z-z_{c}\right)\right\|$.

Proof: For details please see [45].

As stated in the Introduction, reward/cost shaping has been developed including barrier functions of constraints in cost function (2), see [41]-[44]. However, such a design fails to guide how to guarantee safety by an actor-critic structure design. Consequently, the abrupt cost function changes due to the safety-related terms in approaching the constraint boundary could cause the divergence of the weights of the actorcritic networks in the training process. Also, few works have addressed the safe RL algorithm design under time-varying safety constraints. For this reason, we propose a safe RL approach using a BCP structure and an MPE mechanism for optimal control under time-varying safety constraints. Specifically, we present a safe actor-critic structure for implementing the proposed safe RL, enabling actor-critic learning with safety and convergence guarantees.

\section{SAFE RL WITH BCP AND MPE}

This section presents our safe RL approach and its implementation by a barrier-based actor-critic learning algorithm.

\section{A. Design of safe RL with $B C P$ and $M P E$}

We reconstruct the performance index $J\left(x_{k}\right)$ with state and control barrier functions defined in (4). Letting $\mu>0$ be a tuning parameter, the resulting value function, denoted as $\bar{J}\left(x_{k}\right)$, is defined as

$$
\bar{J}\left(x_{k}\right)=\sum_{k=0}^{+\infty} \gamma^{k} \bar{r}\left(x_{k}, u_{k}\right)
$$

where $\bar{r}\left(x_{k}, u_{k}\right)=r\left(x_{k}, u_{k}\right)+\mu \mathcal{B}_{k}\left(u_{k}\right)+\mu \mathcal{B}_{k}\left(x_{k}\right)$.
Remark 1: Barrier functions have been recently used to cope with state constraints in RL algorithms, see e.g. [29], [46], [47]. The main idea is to replace the state variable with a virtual one using a special invertible barrier function. In our case, this invertible restriction is relaxed by (11) that $\mathcal{B}(\cdot)$ is differentiable. Crucially, a different stabilizing control problem rather than an optimal control one is considered in [29], [46], [47].

Let at any time instant $k$, the $L$-step ahead control policy be $u\left(x_{\tau}\right) \forall \tau \in \mathbb{N}_{0}^{L-1}$ where $L \in \mathbb{N}$. Hence, one can write the following difference equation for the multi-step prediction of the stage cost under $u(x)$, i.e.,

$$
\begin{aligned}
\bar{J}\left(x_{k}\right) & =\bar{r}\left(x_{k}, u\left(x_{k}\right)\right)+\gamma \bar{J}\left(x_{k+1}\right) \\
& =\sum_{l=0}^{L-1} \gamma^{l} \bar{r}\left(x_{k+l}, u\left(x_{k+l}\right)\right)+\gamma^{L} \bar{J}\left(x_{k+L}\right) .
\end{aligned}
$$

Letting $s_{k}\left(x_{k}\right)=\sqrt{\mathcal{B}_{k}\left(x_{k}\right)}$, the control problem for (1) with cost (7) is equivalent to an unconstrained optimal control problem for the system

$$
\left\{\begin{array}{l}
x_{k+1}=f\left(x_{k}, u_{k}\right) \\
y_{k}=\left(x_{k}, s_{k}\left(x_{k}\right)\right)
\end{array}\right.
$$

with cost $\bar{J}$ being rewritten as

$$
\bar{J}\left(x_{k}\right)=\sum_{k=0}^{+\infty} \gamma^{k}\left(\left\|y_{k}\right\|_{Q_{y}}^{2}+\left\|u_{k}\right\|_{R}^{2}+\mu \mathcal{B}_{k}\left(u_{k}\right)\right),
$$

where $Q_{y}=\operatorname{diag}\{Q, \mu\}$.

In (10), the overall objective function consists of the classical quadratic-type regulation costs and the barrier functions on the state and control. The tuning parameter $\mu$ determines the influence of the barrier function values on the overall objective function. Given $\mu$, the barrier functions even become dominant if the control and state are close to the boundaries of the safety constraints. Indeed, the parameter $\mu$ (with respect to $Q$ and $R$ ) represents a trade-off between optimality and learning safety.

To solve the control problem with (9) and (10), we propose a novel type of control policy as follows:

$$
u_{k}=v_{k}+\rho \nabla_{v} \mathcal{B}_{k}\left(v_{k}\right)+K \nabla_{x} \mathcal{B}_{k}\left(x_{k}\right)
$$

where $v_{k} \in \mathbb{R}^{m}$ is a new virtual control input, $\rho \in \mathbb{R}$ and $K \in \mathbb{R}^{m \times n}$ are decision variables to be further optimized (see also Section IV); $\nabla_{v} \mathcal{B}_{k}\left(v_{k}\right)$ is the gradient of $\mathcal{B}_{k}\left(v_{k}\right)$ for $v_{k} \in \mathcal{U}_{k}, \nabla_{x} \mathcal{B}_{k}\left(x_{k}\right)$ is the gradient of $\mathcal{B}_{k}\left(x_{k}\right)$ for $x_{k} \in \mathcal{X}_{k}$.

Remark 2: In (11), the roles of the second and third terms are to generate the repulsive forces, respectively, as the states $x$ and $v$ move toward the corresponding boundary of the constraints. As such, the state and control can be restrained in their interiors. That is to say, safety is guaranteed by the gradients of the barrier functions of state and control constraints in (11), any of which will go to infinity (or sufficiently large with the relaxed barrier functions) as the corresponding variable reaches the boundary. In a particular unconstrained case, the control policy is reduced to $u\left(x_{k}\right)=v\left(x_{k}\right)$, which is the same type used in the classic RL algorithms [48]-[50]. 
Under control (11), letting $\bar{J}^{*}\left(x_{k}\right)$ be the optimal value function at time instant $k$, a variant of the discrete-time HJB equation can be written as

$$
\begin{aligned}
\bar{J}^{*}\left(x_{k}\right) & =\min _{u_{k} \in \mathcal{U}_{k}} \bar{r}\left(x_{k}, u\left(x_{k}\right)\right)+\gamma \bar{J}^{*}\left(x_{k+1}\right) \\
& =\min _{u_{k+l} \in \mathcal{U}_{k+l}, l \in \mathbb{N}_{0}^{L-1}} \sum_{l=0}^{L-1} \gamma^{l} \bar{r}\left(x_{k+l}, u\left(x_{k+l}\right)\right)+\gamma^{L} \bar{J}^{*}\left(x_{k+L}\right)
\end{aligned}
$$

and the optimal solution is

$$
\begin{aligned}
u^{*}\left(x_{k}\right) & =\underset{u_{k} \in \mathcal{U}_{k}}{\operatorname{argmin}} \bar{r}\left(x_{k}, u\left(x_{k}\right)\right)+\gamma \bar{J}^{*}\left(x_{k+1}\right) \\
& =\underset{u_{k+l} \in \mathcal{U}_{k+l}, l \in \mathbb{N}_{0}^{L-1}}{\operatorname{argmin}} \sum_{l=0}^{L-1} \gamma^{l} \bar{r}\left(x_{k+l}, u\left(x_{k+l}\right)\right)+\gamma^{L} \bar{J}^{*}\left(x_{k+L}\right)
\end{aligned}
$$

We propose a safe RL algorithm with barrier-based control policy (BCP) and multi-step policy evaluation (MPE) in Algorithm 1 to solve $u^{*}\left(x_{k}\right)$ and $\bar{J}^{*}\left(x_{k}\right)$.

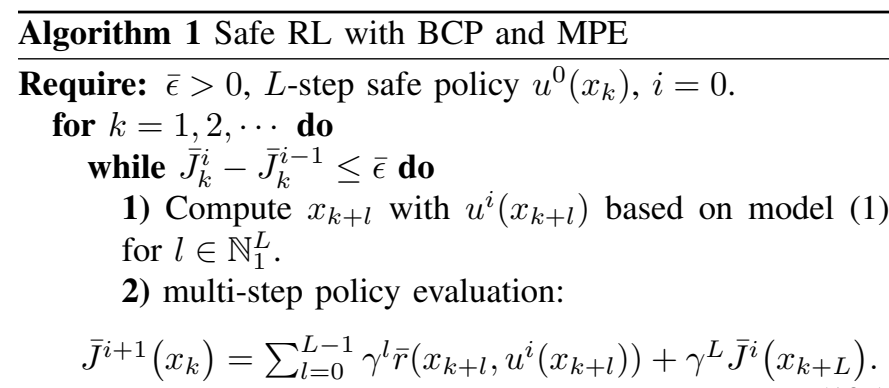

3) Barrier-based control policy update:

$$
\begin{gathered}
\left(v\left(x_{k}\right), \rho, K\right)^{i+1}=\underset{v_{k}, \rho, K}{\operatorname{argmin}} \bar{r}\left(x_{k}, u\left(x_{k}\right)\right)+\gamma \bar{J}^{i+1}\left(x_{k+1}\right), \\
u^{i+1}\left(x_{k}\right)=v^{i+1}\left(x_{k}\right)+\rho^{i+1} \nabla_{v} \mathcal{B}_{k}\left(v^{i+1}\left(x_{k}\right)\right) \\
+K^{i+1} \nabla_{x} \mathcal{B}_{k}\left(x_{k}\right) .
\end{gathered}
$$

$$
\begin{aligned}
& \text { 4) } i \leftarrow i+1 . \\
& \text { end while } \\
& \text { end for }
\end{aligned}
$$

Remark 3: Different from cost shaping-based RL algorithms [10], [41]-[44], [46] and primal-dual approaches [15], [37]-[39], in the proposed approach, the decision variables $v\left(x_{k}\right), \rho$, and $K$, instead of $u\left(x_{k}\right)$, are optimized in (12b). Then the improved decision variables are used to compute the real control policy $u\left(x_{k}\right)$. The potential benefit lies in a clear mechanistic interpretation of the safety guarantee mechanism (see (11)) and the inspiration of a consistent, barrier-based actor-critic structure for implementing Algorithm 1 (see Section III-B).

Remark 4: Note that model-free RL algorithms have received considerable attention in continuous control tasks [3]. However, model-free approaches still have the data-inefficient issue, suitable for specific tasks with valid datasets [16], [51]. In our case, we focus on a model-based framework with safety and convergence guarantees because it is more flexible to our concerned real-world safety-critical vehicle control tasks. The extension of our approach to the model-free case will be left for further investigation.

\section{B. Barrier-based actor-critic learning for safe $R L$}

In the following, Algorithm 1 is implemented with a barrierbased actor-critic learning structure. We first construct a consistent type of critic network to $\bar{J}$ in (7) with barrier functions:

$$
\hat{\bar{J}}\left(x_{k}\right)=W_{c 1}^{\top} \sigma_{c}\left(x_{k}\right)+W_{c 2} \mathcal{B}_{k}\left(x_{k}\right)
$$

where $W_{c 1} \in \mathbb{R}^{N_{c}}$ and $W_{c 2} \in \mathbb{R}$ are weighting matrices, $\sigma_{c} \in \mathbb{R}^{N_{c}}$ is a vector composed of basis functions. In a collective form, we write $\hat{\bar{J}}\left(x_{k}\right)=W_{c}^{\top} h_{c}\left(x_{k}\right)$, where $W_{c}=\left(W_{c 1}, W_{c 2}\right), h_{c}\left(x_{k}\right)=\left(\sigma_{c}\left(x_{k}\right), \mathcal{B}_{k}\left(x_{k}\right)\right)$.

The ultimate goal of the critic network is to minimize the distance between $\bar{J}^{*}$ and $\hat{\bar{J}}$ via updating $W_{c}$. However, as $\bar{J}^{*}$ is not available, the following $\bar{J}^{d}\left(x_{k}\right)$ (defined according to $(12 \mathrm{a}))$ is used as the target to be steered by $\hat{\bar{J}}$, i.e.,

$$
\bar{J}^{d}\left(x_{k}\right)=\sum_{l=0}^{L-1} \gamma^{l} \bar{r}\left(x_{k+l}, u_{k+l}\right)+\gamma^{L} \hat{\bar{J}}\left(x_{k+L}\right)
$$

Let $\varepsilon_{c, k}=\bar{J}^{d}\left(x_{k}\right)-\hat{\bar{J}}\left(x_{k}\right)$ be the approximation residual, $\delta_{c, k}=\varepsilon_{c, k}^{2}$, and $\gamma_{c}$ be the learning rate, then the update rule of the weighting matrix $W_{c}$ is given as

$$
W_{c, k+1}=W_{c, k}-\gamma_{c} \frac{\partial \delta_{c, k}}{\partial W_{c, k}}
$$

We next design the actor network for learning the control policy (11) with the following form

$$
\left.u\left(x_{k}\right)=W_{a, \sigma}^{\top} \sigma_{a}\left(x_{k}\right)+\hat{K} \nabla_{x} \mathcal{B}_{k}\left(x_{k}\right)\right)+\hat{\rho} \nabla_{v} \mathcal{B}_{k}\left(v_{k}\right)
$$

where $W_{a, \sigma} \in \mathbb{R}^{N_{u} \times m}, \hat{K} \in \mathbb{R}^{m \times n}$, and $\hat{\rho} \in \mathbb{R}$ are the weighting matrices, $\sigma_{a} \in \mathbb{R}^{N_{u}}$ is a vector of basis functions. Let $W_{a}^{\top}=\left[W_{a 1}^{\top} \hat{\rho} I\right], W_{a 1}^{\top}=\left[W_{a, \sigma}^{\top} \hat{K}\right]$ and $h_{a}\left(x_{k}\right)=$ $\left(h_{a 1}\left(x_{k}\right), \nabla_{v} \mathcal{B}_{k}\left(v_{k}\right)\right), h_{a 1}\left(x_{k}\right)=\left(\sigma_{a}\left(x_{k}\right), \nabla_{x} \mathcal{B}_{k}\left(x_{k}\right)\right)$, then one can write (16) in a collective form as $u\left(x_{k}\right)=W_{a}^{\top} h_{a}\left(x_{k}\right)$.

Remark 5: As mentioned in Section I, the classic actor-critic design with a neural network is prone to training divergence due to abrupt cost function changes. To solve this problem, the proposed actor and critic comprise a neural network and safety-related terms multiplied by weighting parameters (see (13) and (16)), where the weights are updated to ensure learning convergence. In the critic (13), the safety-related term is to capture sudden variations of the barrier functions in $\bar{J}\left(x_{k}\right)$. In the actor (16), the safety-related terms have clear mechanistic interpretations for safety certificates (see Remark 3). In principle, an extra neural network might be used for representing the safety-related terms in (13) and (16). However, our theoretical results might not directly hold in this case due to possible approximation errors of neural networks. The extension to this case with theoretical guarantees is an open problem and will be left for further investigation.

As the optimal control $u^{*}$ is unavailable and in view of (12b) and (16), letting $\nu_{k}=2 R u_{k}+\mu \nabla_{u} \mathcal{B}_{k}\left(u_{k}\right)$, we define a desired target of $\nu_{k}$, i.e., $\nu_{k}^{d}$ as $\nu_{k}^{d}=-\nabla_{u} f(x, u)^{\top} \partial \hat{\bar{J}}\left(x_{k+1}\right) / \partial x_{k+1}$. Denote $\varepsilon_{a, k}=\nu_{k}^{d}-\nu_{k}$ as the approximation residual, $\delta_{a, k}=$ $\left\|\varepsilon_{a, k}\right\|^{2}$, and $\gamma_{a}$ be the learning rate, then the update rule of $W_{a 1}$ and $\hat{\rho}$ is given as 


$$
\begin{aligned}
W_{a 1, k+1} & =W_{a 1, k}-\gamma_{a} \frac{\partial \delta_{a, k}}{\partial W_{a 1, k}} \\
\hat{\rho}_{k+1} & =\hat{\rho}_{k}-\gamma_{a} \frac{\partial \delta_{a, k}}{\partial \hat{\rho}_{k}},
\end{aligned}
$$

\section{THEORETICAL RESUlTS}

The theoretical properties of the proposed safe RL in nominal and disturbance scenarios are proven in Section IV-A and Section IV-B respectively. Then, the convergence analysis of the barrier-based actor-critic learning algorithm under timeinvariant constraints is given in Section IV-C.

\section{A. Safety and stability guarantees in nominal scenario}

The convergence of RL with MPE for unconstrained nonlinear systems was proven in [50]. In this note, we prove the safety and convergence of our proposed safe RL with BCP and MPE, i.e., Algorithm 1 under time-varying constraints.

Theorem 1 (Control safety and convergence): If $u^{0}\left(x_{k}\right)=$ $v^{0}\left(x_{k}\right)+\rho^{0} \nabla_{v} \mathcal{B}_{k}\left(v^{0}\left(x_{k}\right)\right)+K^{0} \nabla_{x} \mathcal{B}_{k}\left(x_{k}\right)$ is an $L$-step safe control policy for (1) and the value function $\bar{J}^{0}\left(x_{k}\right) \geq$ $\bar{r}\left(x_{k}, u^{0}\left(x_{k}\right)\right)+\gamma \bar{J}^{0}\left(x_{k+1}\right)$; then with (12), it holds that

1) $\bar{J}^{i+1}\left(x_{k}\right) \leq V^{i}\left(x_{k}\right) \leq \bar{J}^{i}\left(x_{k}\right)$, where $V^{i}\left(x_{k}\right)=$ $\bar{r}\left(x_{k}, u^{i}\left(x_{k}\right)\right)+\gamma \bar{J}^{i}\left(x_{k+1}\right)$

2) $u^{i}\left(x_{k}\right)=v^{i}\left(x_{k}\right)+\rho^{i} \nabla_{v} \mathcal{B}_{k}\left(v^{i}\left(x_{k}\right)\right)+K^{i} \nabla_{x} \mathcal{B}_{k}\left(x_{k}\right)$ is a $L$-step safe control policy;

3) $\bar{J}^{i}\left(x_{k}\right) \rightarrow \bar{J}^{*}\left(x_{k}\right)$ and $u^{i}\left(x_{k}\right) \rightarrow u^{*}\left(x_{k}\right)$, as $i \rightarrow+\infty$.

Proof. 1): First note that,

$$
\begin{aligned}
\bar{J}^{1}\left(x_{k}\right)= & \sum_{l=0}^{L-1} \gamma^{l} \bar{r}\left(x_{k+l}, u^{0}\left(x_{k+l}\right)\right)+\gamma^{L} \bar{J}^{0}\left(x_{k+L}\right) \\
= & \sum_{l=0}^{L-2} \gamma^{l} \bar{r}\left(x_{k+l}, u^{0}\left(x_{k+l}\right)\right)+ \\
& \gamma^{L-1} \bar{r}\left(x_{k+L-1}, u^{0}\left(x_{k+L-1}\right)\right)+\gamma^{L} \bar{J}^{0}\left(x_{k+L}\right) \\
= & \sum_{l=0}^{L-2} \gamma^{l} \bar{r}\left(x_{k+l}, u^{0}\left(x_{k+l}\right)\right)+\min _{\left\{v_{k+L-1}, \rho, K\right\}} \gamma^{L-1}\{ \\
& \left.\bar{r}\left(x_{k+L-1}, u\left(x_{k+L-1}\right)\right)+J^{0}\left(x_{k+L}\right)\right\} \\
= & \sum_{l=0}^{L-2} \gamma^{l} \bar{r}\left(x_{k+l}, u^{0}\left(x_{k+l}\right)\right)+ \\
\leq & \sum_{l=0}^{L-2} \gamma^{l} \bar{r}\left(x_{k+l}, u^{0}\left(x_{k+l}\right)\right)+\gamma^{L-1} \bar{J}^{0}\left(x_{k+L-1}\right) \\
\vdots & \\
\leq & \bar{r}\left(x_{k}, u^{0}\left(x_{k}\right)\right)+\gamma \bar{J}^{0}\left(x_{k+1}\right)=V^{0}\left(x_{k}\right) \leq \bar{J}^{0}\left(x_{k}\right) .
\end{aligned}
$$

Hence, $\bar{J}^{1}\left(x_{k}\right) \leq V^{0}\left(x_{k}\right) \leq \bar{J}^{0}\left(x_{k}\right)$. Then one can obtain the result by induction.

2): Since $u^{0}\left(x_{k}\right)$ is an $L$-step safe policy, and $\bar{J}^{0}\left(x_{k}\right)<$ $+\infty$, in view of Claim 1), it holds that $\bar{J}^{1}\left(x_{k}\right) \leq \bar{J}^{0}\left(x_{k}\right)<$ $+\infty$. As a consequence, $\sum_{l=0}^{L-1} \gamma^{l} \bar{r}\left(x_{k+l}, u^{0}\left(x_{k+l}\right)\right)<+\infty$, leading to $\mathcal{B}_{k+l}\left(u_{k+l}^{1}\right), \mathcal{B}_{k+l}\left(x_{k+l}\right)<+\infty$ for all $l \in \mathbb{N}_{1}^{L}$. That is to say, $u_{\tau}^{1} \in \mathcal{U}_{\tau}$ and $x_{\tau} \in \mathcal{X}_{\tau}$ for all $\tau \in[k, k+$ $L]$. Hence, $u^{1}\left(x_{k}\right)$ is an $L$-step safe policy. Then, one can summarize by induction that $u^{i}\left(x_{k}\right)$ is $L$-step safe $\forall i>0$.

3): Since $\bar{J}^{i+1}\left(x_{k}\right) \leq \bar{J}^{i}\left(x_{k}\right)$ and $\bar{J}^{i}\left(x_{k}\right)$ is a semidefinite positive function in view of the property of the barrier function. Then one can conclude that $\bar{J}^{i}\left(x_{k}\right)$ converges to a value denoted as $\bar{J}^{\infty}\left(x_{k}\right) \geq 0$. From Claim 1), one has $\bar{J}^{\infty}\left(x_{k}\right) \leq V^{\infty}\left(x_{k}\right) \leq \bar{J}^{\infty}\left(x_{k}\right)$, then

$$
\begin{aligned}
& \bar{J}^{\infty}\left(x_{k}\right)=V^{\infty}\left(x_{k}\right) \\
& =\min _{\left\{v_{k}, \rho, K\right\}} \bar{r}\left(x_{k}, u\left(x_{k}\right)\right)+\gamma \bar{J}^{\infty}\left(x_{k+1}\right) \\
& =\min _{\left\{v_{k+i}, \rho, K\right\}, i \in \mathbb{N}_{0}^{1}} \sum_{l=0}^{1} \gamma^{l} \bar{r}\left(x_{k+l}, u\left(x_{k+l}\right)\right)+\gamma^{2} \bar{J}^{\infty}\left(x_{k+2}\right) \\
& =\min _{\left\{v_{k+i}, \rho, K\right\}, i \in \mathbb{N}_{0}^{L-1}} \sum_{l=0}^{L-1} \gamma^{l} \bar{r}\left(x_{k+l}, u\left(x_{k+l}\right)\right)+\gamma^{L} \bar{J}^{\infty}\left(x_{k+L}\right) \\
& =\min _{\left\{v_{k+i}, \rho, K\right\}, i \in \mathbb{N}} \bar{J}^{\infty}\left(x_{k}\right)=\bar{J}^{*}\left(x_{k}\right)
\end{aligned}
$$

One can promptly conclude that, $\bar{J}^{\infty}\left(x_{k}\right)=\bar{J}^{*}\left(x_{k}\right)$. And $v^{\infty}, \rho^{\infty}$, and $K^{\infty}$ equal to the optimal values $v^{*}\left(x_{k}\right), \rho^{*}$, and $K^{*}$, respectively. Consequently, $u^{\infty}\left(x_{k}\right)=v^{\infty}\left(x_{k}\right)+$ $\rho^{\infty} \nabla_{v} \mathcal{B}_{k}\left(v^{\infty}\left(x_{k}\right)\right)+K^{\infty} \nabla_{x} \mathcal{B}_{k}\left(x_{k}\right)=u^{*}\left(x_{k}\right)$.

Assumption 4 (Stabilizability): There exist $v\left(x_{k}\right), \rho, K$ such that (9) is stabilizable under (11).

Proposition 1 (Stability): Let $\gamma=1, x_{0} \in \mathcal{X}_{0}$, and $u^{*}\left(x_{k}\right)$ be obtained with the optimal solution $v^{*}\left(x_{k}\right), \rho^{*}$, and $K^{*}$, solved via minimizing (10) with (9). Under Assumptions 1 and 4 , the state $x_{k}$ of model (1) using $u^{*}\left(x_{k}\right)$, converges to the origin as $k \rightarrow+\infty$.

Proof. In view of (5) and (1), one can observe that $u_{k}=0$ for $k \geq \bar{k}$ provided that $v_{k}=0$ and $x_{k}=0$. Also, $\mathcal{B}_{k}\left(u_{k}\right)=$ 0 as $u_{k}=0$ and $s_{k}=0$ as $x_{k}=0$ for $k \geq \bar{k}$. Hence, $\bar{J}^{*}\left(x_{k}\right)<+\infty$ under $u^{*}\left(x_{k}\right)$ with $v^{*}\left(x_{k}\right), \rho^{*}, K^{*}$. As a result, $y_{k}, u_{k} \rightarrow 0$ as $k \rightarrow+\infty$ under policy $u^{*}\left(x_{k}\right)$. Consequently, $x_{k} \rightarrow 0$ as $k \rightarrow+\infty$.

Remark 6: Note that the discount factor $0<\gamma<1$ is a crucial ingredient in reinforcement learning to ensure the convergence of the value function and control policy to the optimal ones, see [52] and condition (21b). However, as illustrated by Proposition 1, a choice of $\gamma$ close to 1 is suggested to guarantee closed-loop stability.

\section{B. Safety and robustness guarantees in disturbed scenario}

We show that our approach can guarantee safety and robustness under disturbances by properly shrinking the state constraints in the learning process. To this end, let the real model be given as

$$
z_{k+1}=f\left(z_{k}, u_{k}\right)+w_{k},
$$

where $z_{k}$ is the real state, $w_{k} \in \mathcal{W}$ is an additive bounded and unknown disturbance which can represent the modeled uncertainty or measurement noise. In case the model dynamics are unavailable, the derivation of the nominal model (1) may resort to data-driven modeling approaches. For a specific datadriven modeling approach and the estimation of the associated uncertainty set $\mathcal{W}$ please refer to [40].

Let at any time instant $k, x_{k+j \mid k}$ be the predicted state by applying the control $u\left(x_{k}\right), \cdots, u\left(x_{k+L-1}\right)$ using model (1). Assuming that the uncertainty set $\mathcal{W}$ is norm-bounded, i.e., $\left\|w_{k}\right\| \leq \varepsilon_{w}$, then the following lemma is stated.

Lemma 2 ([53]): The difference between the real state under $u(z)$ and the nominal one under $u(x)$ satisfies

$$
\left\|x_{k+j \mid k}-z_{k+j}\right\| \leq \frac{L_{f}^{j}-1}{L_{f}-1} \varepsilon_{w}
$$


where $x_{k \mid k}=z_{k}$.

Proof. The proof is similar to [53].

Let the constraint on the nominal state be shrunken, i.e., $x_{k+j \mid k} \in \overline{\mathcal{X}}_{k+j}$ where $\overline{\mathcal{X}}_{k+j}=\mathcal{X}_{k+j} \ominus \mathcal{D}_{\varepsilon_{w}}^{j}, \mathcal{D}_{\varepsilon_{w}}^{j}=\{y \in$ $\left.\mathbb{R}^{n} \mid\|y\| \leq \frac{L_{f}^{j}-1}{L_{f}-1} \varepsilon_{w}\right\}$. The barrier function on the state in (7) is modified according to the constraint $x_{k+j \mid k} \in \overline{\mathcal{X}}_{k+j}$. Assume that the computed $\overline{\mathcal{X}}_{k+j}$ is non-empty and contains the origin in the interior for all $k \geq \bar{k}$.

Theorem 2 (Control safety and robustness): Under Assumptions 1-4, the state evolution of (19), by applying the offline learned policy $u^{*}$ with (1), converges to the set $\mathcal{D}_{\varepsilon_{w}}^{\infty}$, i.e., $\lim _{k \rightarrow+\infty} x_{k} \rightarrow \mathcal{D}_{\varepsilon_{w}}^{\infty}$.

Proof. (i) Offline learning scenario. In view of the Lipschitz continuity condition (3), the difference of the real state $z$ under $u^{*}(z)$ and the nominal state $x$ under $u^{*}(x)$ can be computed as $\left\|z_{1}-x_{1 \mid 0}\right\|=\left\|w_{0}\right\| \leq \epsilon_{w}$, since $x_{0 \mid 0}=z_{0}$. Then, by induction, one has

$$
\left\|z_{j}-x_{j \mid 0}\right\| \leq\left\|z_{j-1}-x_{j-1 \mid 0}\right\|+\epsilon_{w} \leq \frac{L_{f}^{j}-1}{L_{f}-1} \varepsilon_{w} .
$$

Hence, the real state $z_{k}$ converges to $\mathcal{D}_{\varepsilon_{w}}^{\infty}$ as $k \rightarrow+\infty$.

(ii) Online learning scenario. Given an offline learned control policy, at any time instant $k$, it is learned to obtain an improved control performance under the constraint $x_{k+j \mid k} \in \overline{\mathcal{X}}_{k+j}$. In this case, the control policy can not be updated if the learned one is evaluated (by MPE) to be inferior to the current one. Given the proof in the offline learning case, the real state of the online learned policy converges to the set $\mathcal{D}_{\varepsilon_{w}}^{\infty}$.

As suggested in [53], to reduce the size of $\mathcal{D}_{\varepsilon_{w}}^{j}$, i.e., the Lipschitz constant $L_{f}$, two design choices are suggested: (i) a different suitable norm type can be used; (ii) an additional feedback term $K\left(z_{k}-x_{k}\right)$ can be added in the control input to reduce the conservativeness of the multi-step prediction of (1), where $K \in \mathbb{R}^{m \times n}$ is a stabilizing gain matrix of (1).

\section{Convergence analysis of BAC learning algorithm}

In the following, we prove the convergence of the barrierbased actor-critic learning algorithm using the Lyapunov method. The proof is completed using the nominal model under the assumption that state and control constraints are time-invariant, i.e., $\mathcal{X}=\mathcal{X}_{k}$ and $\mathcal{U}=\mathcal{U}_{k}$. Also for simplicity, we assume that $G^{i}(u)=E^{i} u, E^{i} \in \mathbb{R}^{1 \times m}$. First, one write

$$
\begin{gathered}
\bar{J}^{*}\left(x_{k}\right)=W_{c}^{* \top} h_{c}\left(x_{k}\right)+\kappa_{c}\left(x_{k}\right) \\
u^{*}\left(x_{k}\right)=W_{a}^{* \top} h_{a}\left(x_{k}\right)+\kappa_{a}\left(x_{k}\right),
\end{gathered}
$$

where $W_{c}^{*}$ and $W_{a}^{*}$ are constant weights, $\kappa_{c}$ and $\kappa_{a}$ are reconstruction errors. We require the following assumption about the actor and critic network.

Assumption 5 (Weights and reconstruction errors of $B A C$ ):

1) $\left\|W_{c}^{*}\right\| \leq W_{c, m},\left\|\sigma_{c}(x)\right\| \leq \sigma_{c, m},\left\|\nabla_{x} \sigma_{c}(x)\right\| \leq \bar{\sigma}_{c, m}$, $\left\|\kappa_{c}(x)\right\| \leq \kappa_{c, m}$

2) $\left\|W_{a}^{*}\right\| \leq W_{a, m},\left\|\sigma_{a}(x)\right\| \leq \sigma_{a, m},\left\|\kappa_{a}(x)\right\| \leq \kappa_{a, m}$.

To state the following theorem in a compact form, we let $\tilde{W}_{\star}=W_{\star}^{*}-W_{\star}$, $=a, c$ in turns, denote $\Delta \bar{h}_{c, k}=$ $\Delta h_{c, k}^{\top} \Delta h_{c, k}$, where $\Delta h_{c, k}=\gamma^{L} h_{c, k+L}-h_{c, k}$, and use $q$ and $q^{+}$to denote $q_{k}$ and $q_{k+1}$ respectively unless otherwise specified.

Theorem 3 (Convergence of BAC learning): Under Assumptions 3 and 5 , if

$$
R-\mu H_{u} \succ 0 \text { and } I-3 d_{m}\left(R+\mu H_{u}\right)^{2} \succ 0
$$

where $d_{m}=4 \gamma_{a}\left(\sigma_{a, m}^{2}+\mathcal{B}_{v, m}^{2}+\mathcal{B}_{x, m}^{2}\right)$, and

$$
q_{1} \leq \Delta \bar{h}_{c, k} \leq q_{2}
$$

where $q_{1}, q_{2}>0$, then it holds that $\left\|\left(\xi_{a, k}, \tilde{W}_{c, k}\right)\right\| \leq$ $\sqrt{\frac{\epsilon_{m}}{\lambda_{\min }(S)}}$, as $k \rightarrow+\infty$, where $\xi_{a, k}=\tilde{W}_{a, k}^{\top} h_{a}\left(x_{k}\right), \epsilon_{m}$ is a bounded error and $S$ is a positive-definite matrix, which are deferred in (34) and (35). Also, $\left(\xi_{a, k}, \tilde{W}_{c, k}\right) \rightarrow 0$, as $k \rightarrow+\infty$, if $\kappa_{\star}\left(x_{k}\right) \rightarrow 0, \star=a, c$ in turns.

Proof. Define a Lyapunov function

$$
V_{k}=\underbrace{\alpha_{c} / \gamma_{c} \operatorname{tr}\left(\tilde{W}_{c, k}^{\top} \tilde{W}_{c, k}\right)}_{\alpha_{c} V_{c, k}}+\underbrace{1 / \gamma_{a} \operatorname{tr}\left(\tilde{W}_{a, k}^{\top} \tilde{W}_{a, k}\right)}_{V_{a, k}}
$$

where $\alpha_{c}>1$. In view of the update rule (15) and (17), one can write the difference of $V_{\star, k}$ ( $\star=a, c$ in turns), i.e., $\Delta V_{\star, k}=V_{\star, k+1}-V_{\star, k}$ as

$$
\Delta V_{\star, k}=\operatorname{tr}\left(2 \tilde{W}_{\star, k}^{\top} \frac{\partial \delta_{\star, k}}{\partial W_{\star, k}}+\gamma_{\star}\left(\frac{\partial \delta_{\star, k}}{\partial W_{\star, k}}\right)^{\top} \frac{\partial \delta_{\star, k}}{\partial W_{\star, k}}\right)
$$

To first compute $\Delta V_{c}$, note that

$$
\frac{\partial \delta_{c}}{\partial W_{c}}=\frac{\partial<\varepsilon_{c}, \varepsilon_{c}>}{\partial W_{c}}=2 \Delta h_{c} \varepsilon_{c}^{\top} .
$$

Moreover,

$$
\begin{aligned}
& \varepsilon_{c, k}=\sum_{l=0}^{L-1} \gamma^{l} \bar{r}\left(x_{k+l}, u_{k+l}\right)+\gamma^{L} \hat{\bar{J}}\left(x_{k+L}\right)-\hat{\bar{J}}\left(x_{k}\right) \\
& =-\gamma^{L} \bar{J}^{*}\left(x_{k+L}\right)+\bar{J}^{*}\left(x_{k}\right)+\gamma^{L} \hat{\bar{J}}\left(x_{k+L}\right)-\hat{\bar{J}}\left(x_{k}\right) \\
& =-\tilde{W}_{c, k}^{\top} \Delta h_{c, k}-\Delta \kappa_{c, k}
\end{aligned}
$$

where $\Delta \kappa_{c, k}=\gamma^{L} \kappa_{c, k+L}-\kappa_{c, k}$, the second equality in (25) is due to the Bellman equation (8).

Taking (24) with (25) into (23), leads to

$$
\begin{aligned}
\Delta V_{c} & =-4 c_{1}\left\|\tilde{W}_{c}\right\|^{2}+4 \Delta \kappa_{c} c_{2} \tilde{W}_{c}+4 \gamma_{c} \Delta \bar{h}_{c} \Delta \kappa_{c}^{2} \\
& \leq-4\left(c_{1}-\lambda_{\max }\left(\bar{c}_{2}\right) \beta_{1}\right)\left\|\tilde{W}_{c}\right\|^{2}+\epsilon_{\kappa, 1}
\end{aligned}
$$

where $c_{1}=\left(1-\gamma_{c} \Delta \bar{h}_{c}\right) \Delta \bar{h}_{c}, c_{2}=-\left(1-2 \gamma_{c} \Delta \bar{h}_{c}\right) \Delta h_{c}^{\top}$, $\bar{c}_{2}=c_{2}^{\top} c_{2}, \epsilon_{\kappa, 1}=4\left(\gamma_{c} \Delta \bar{h}_{c}+1 / \beta_{1}\right) \Delta \kappa_{c}^{2}, \beta_{1}$ is a tuning constant.

To compute $\Delta V_{a}$, inline with (24), one has

$$
\frac{\partial \delta_{a}}{\partial \hat{\rho}}=-2 \nabla \mathcal{B}(v) \varepsilon_{a}^{\top} \bar{R}
$$

where $\bar{R}=2 R+\mu \nabla^{2} \mathcal{B}(u), \nabla \mathcal{B}(z)$ stands for $\nabla_{z} \mathcal{B}(v)$ for a general variable $z$; and

$$
\frac{\partial \delta_{a}}{\partial W_{a 1}}=-2 h_{a 1} \varepsilon_{a}^{\top} \bar{R} .
$$

With (27), we write $\Delta V_{a}=\Delta V_{a 1}+\Delta V_{a 2}$ where $\Delta V_{a 1}$ and $\Delta V_{a 2}$ are given as

$$
\begin{aligned}
\Delta V_{a 1} & =4 \operatorname{tr}\left(-\tilde{W}_{a 1}^{\top} h_{a 1} \varepsilon_{a}^{\top} \bar{R}+\gamma_{a} \bar{R}^{\top} \varepsilon_{a} \bar{h}_{a 1} \varepsilon_{a}^{\top} \bar{R}\right) \\
\Delta V_{a 2} & =4 \operatorname{tr}\left(-\tilde{\rho} \nabla \mathcal{B}(v) \varepsilon_{a}^{\top} \bar{R}+\gamma_{a} \bar{R}^{\top} \varepsilon_{a} \overline{\mathcal{B}}_{v} \varepsilon_{a}^{\top} \bar{R}\right),
\end{aligned}
$$


where $\tilde{\rho}=\rho-\hat{\rho}, \bar{h}_{a 1}=h_{a 1}^{\top} h_{a 1}, \overline{\mathcal{B}}_{v}=\nabla \mathcal{B}(v)^{\top} \nabla \mathcal{B}(v)$. In view of (28), letting $\bar{h}_{a}=\bar{h}_{a 1}+\overline{\mathcal{B}}_{v}$, one can write $\Delta V_{a}$ as

$$
\begin{aligned}
\Delta V_{a} & =\Delta V_{a 1}+\Delta V_{a 2} \\
& =-4 \operatorname{tr}\left(\xi_{a} \varepsilon_{a}^{\top} \bar{R}\right)+4 \gamma_{a} \operatorname{tr}\left(\bar{R}^{\top} \varepsilon_{a} \bar{h}_{a} \varepsilon_{a}^{\top} \bar{R}\right) .
\end{aligned}
$$

Let $g_{c}=\nabla_{u} f(x, u)^{\top}\left(\nabla h_{c}^{+}\right)^{\top}, \bar{\kappa}_{1}=\nabla_{u} f(x, u)^{\top} \nabla \kappa_{c}^{+}+2 R \kappa_{a}$, then

$$
\begin{aligned}
\varepsilon_{a}= & \nu^{d}-\nu^{*}+\nu^{*}-\nu \\
= & \nu^{d}+g_{c} W_{c}^{*}+\bar{\kappa}_{1}+2 R\left(W_{a}^{*}\right)^{\top} h_{a}+ \\
& \mu \nabla \mathcal{B}\left(u^{*}\right)-2 R W_{a}^{\top} h_{a}-\mu \nabla \mathcal{B}(u)
\end{aligned}
$$

In view of the definition of $\mathcal{B}$ and $G^{i}(u)=E^{i} u$, it holds that

$$
\overline{\mathcal{B}}=\nabla \mathcal{B}\left(u^{*}\right)-\nabla \mathcal{B}(u)=-D\left(\xi_{a}+\kappa_{a}\right)
$$

where $D=\sum_{i=1}^{p_{u}}\left(E^{i}\right)^{\top} E^{i} /\left(G^{i}\left(u^{*}\right) G^{i}(u)\right)$ for $\bar{\sigma} \geq \kappa_{b}$ and $D=2 H_{u}$ otherwise. Hence, with (30), one has

$$
\varepsilon_{a}=g_{c} \tilde{W}_{c}+R_{d} \xi_{a}+\bar{\kappa} .
$$

where $R_{d}=2 R-\mu D$ and $\bar{\kappa}=\bar{\kappa}_{1}-\mu D \kappa_{a}$. Taking (31) into (29), one can compute:

$$
\Delta V_{a}=4\left(-\left\|\xi_{a}\right\|_{R_{d} \bar{R}}^{2}-\xi_{a}^{\top} \bar{R} g_{c} \tilde{W}_{c}-\xi_{a}^{\top} \bar{R} \bar{\kappa}\right)+d\left\|\varepsilon_{a}\right\|_{\bar{R}^{2}}^{2}
$$

where $d=4 \gamma_{a} \bar{h}_{a}$. Applying Young's inequality to (31), leads to $\left\|\varepsilon_{a}\right\|^{2} \leq 3\left\|\tilde{W}_{c}\right\|_{\bar{g}_{c}}^{2}+3\left\|\xi_{a}\right\|_{R_{d}^{2}}^{2}+3\|\bar{\kappa}\|^{2}$, where $\bar{g}_{c}=g_{c}^{\top} g_{c}$. Hence, one can conclude from (32) that

$$
\Delta V_{a} \leq-\left\|\xi_{a}\right\|_{P}^{2}+\left(4 / \beta_{2}+3 d\right) \lambda_{\max }\left(\bar{g}_{c}\right)\left\|\tilde{W}_{c}\right\|^{2}+\epsilon_{\kappa, 2},
$$

where $P=4 R_{d} \bar{R}-\left(\beta_{2}+\beta_{3}\right) \bar{R}^{2}-3 d R_{d}^{2} \bar{R}^{2}, \beta_{2}, \beta_{3}$ are tuning constants, $\epsilon_{\kappa, 2}=\left(4 / \beta_{3}+3 d\right)\|\bar{\kappa}\|_{\bar{R}^{2}}^{2}$. Therefore, combining (26) and (33), it holds that

$$
\Delta V \leq-\left\|\left(\xi_{a}, \tilde{W}_{c}\right)\right\|_{S}^{2}+\epsilon_{t}
$$

where $\epsilon_{t}=\alpha_{c} \epsilon_{\kappa, 1}+\epsilon_{\kappa, 2}$, matrix $S$ is given as

$$
S=\operatorname{diag}\left\{P, 4 \alpha_{c}\left(c_{1}-\bar{c}_{2} \beta_{1}\right)-\left(4 / \beta_{2}+3 d\right) \lambda_{\max }\left(\bar{g}_{c}\right)\right\} .
$$

Note that, in view of Assumption 3 and Lemma 1, $\lambda_{\max }\left(\bar{g}_{c}\right)$ is bounded by $\lambda_{\max }\left(\bar{g}_{c}\right) \leq\left\|\nabla_{u} f(x, u)^{\top}\left(\nabla h_{c}^{+}\right)\right\|^{2} \leq g_{m}^{2}\left(\bar{\sigma}_{c, m}^{2}+\right.$ $\left.\mathcal{B}_{x, m}^{2}\right), R_{d}, \bar{R} \preccurlyeq 2 R+2 \mu H_{u}$, and $d$ can be made small by tuing $\gamma_{a}$, i.e., $d \leq 4 \gamma_{a}\left\|h_{a}\right\|^{2} \leq 4 \gamma_{a}\left(\sigma_{a, m}^{2}+\mathcal{B}_{v, m}^{2}+\mathcal{B}_{x, m}^{2}\right):=$ $d_{m}$. Hence, thanks to (21a), one can first tune $\beta_{2}$ and $\beta_{3}$ such that $P \succ 0$. Then, in view of (21b), one can tune $\alpha_{c}$ and $\beta_{1}$ to make sure the second term of (35) is positive-definite.

Since $\epsilon_{t}$ is bounded, let $\epsilon_{m}$ be the lower bound of $\epsilon_{t}$; then in view of Assumption 5, with $S \succ 0$, it follows that $\Delta V \leq 0$ for all $\left\|\left(\xi_{a, k}, \tilde{W}_{c, k}\right)\right\| \geq \sqrt{\frac{\epsilon_{m}}{\lambda_{\min }(S)}}$. Consequently, $\left\|\left(\xi_{a, k}, \tilde{W}_{c, k}\right)\right\| \rightarrow 0$, as $k \rightarrow+\infty$, provided that $\epsilon_{t} \rightarrow 0$.

\section{Simulation AND EXPERIMENTAL RESUlts}

The developed theoretical results are first verified with two robot simulated examples. Please see the appendix for the detailed implementation steps and results. In this section, we focus on the applications of our approach to two real-world intelligent vehicles. Peculiarly, an integrated path following and collision avoidance problem is considered, which represents a crucial capability for navigation of intelligent vehicles under unknown and dynamic environments [54], [55].

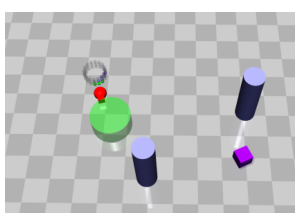

(a)

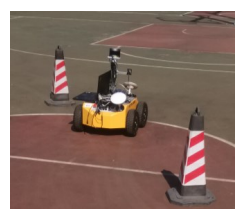

(b)
Fig. 1. (a) Simulation scenario in Safety Gym: the objective is to move the vehicle (red) to the green region while avoiding two static obstacles (grey), the moving soft object (purple) is not considered in the controller design; (b) Experimental platform of the differential-drive vehicle and testing scenario.

\section{A. Application to a differential-drive vehicle with kinematics model: offline learning scenario}

Consider the integrated path following and collision avoidance problem of a differential-drive vehicle. Its kinematics model is

$$
\dot{q}=\left(\dot{p}_{x}, \dot{p}_{y}, \dot{\theta}\right)=\left(v_{o} \cos \theta, v_{o} \sin \theta, \omega\right)
$$

where $\left(p_{x}, p_{y}\right)$ is the coordinate of the vehicle in Cartesian frame, $\theta$ is the yaw angle, $u=\left[v_{o}, \omega\right]^{\top}$ is the control input, where $v_{o}$ and $\omega$ are the linear velocity and yaw rate, respectively.

Let us define the path following error as $e=q_{r}-q$, where $q_{r}$ is the reference state. By discretizing (36), one can write the discrete-time counterpart as

$$
\left\{\begin{aligned}
e_{x}(k+1)= & e_{x}(k)+\Delta t\left(\omega(k) e_{y}(k)-v(k)+\right. \\
& \left.v_{o, r}(k) \cos e_{\theta}(k)\right) \\
e_{y}(k+1)= & e_{y}(k)+\Delta t\left(-\omega(k) e_{x}(k)+\right. \\
& \left.+v_{o, r}(k) \sin e_{\theta}(k)\right) \\
e_{\theta}(k+1)= & e_{\theta}(k)+\Delta t\left(\omega_{r}(k)-\omega(k)\right)
\end{aligned}\right.
$$

where $\left(e_{x}, e_{y}, e_{\theta}\right)=: e, v_{o, r}$ and $\omega_{r}$ are the reference inputs, $\Delta t=0.05 s$ is the adopted sampling interval.

In implementation, the constraint for collision avoidance was typically formulated as $\mathcal{X}=\left\{\left(p_{x}, p_{y}\right)\|\|\left(p_{x}, p_{y}\right)-c \| \geq\right.$ $d\}$, where $d$ and $c$ are the radius and center of the obstacle respectively. Also, the size of $\mathcal{X}$ was properly shrunk by increasing $d$ to account for uncertainties. In the training, the penalty matrices were selected as $Q=I, R=0.1, \mu=0.001$. The discount factor $\gamma$ was $\gamma=0.95$. The relaxing factor $\kappa_{b}$ was $\kappa_{b}=0.05$. The step $L$ was chosen as $L=10$. Weights $W_{c}$ and $W_{a}$ were initialized with uniformly random numbers.

Simulation results using Safety Gym environment [56]. We tested our approach in the Safety Gym environment with the MoJoCo simulator [57]. Our method was compared with some of the state-of-the-art safe RL algorithms: constrained policy optimization (CPO) [58], trust region policy optimization with Lagrangian methods (TRPO-L) [56], proximal policy optimization with Lagrangian methods (PPO-L) [56], deep deterministic policy gradient [59] with cost shaping (DDPGCS), and soft actor-critic (SAC) [3] with cost shaping (SACCS). Note that the safety-aware RL [29] was not directly applicable in this case since it is nontrivial to find an invertible barrier function of the obstacle constraint. In the training stage, all the parameter settings of CPO, TRPO-L, and PPO-L were consistent with that in [56]. In DDPG-CS and SACCS, we used same cost function with ours, i.e., (7). We 
TABLE I

NUMERICAL COMPARISONS IN SAFETY GYM WITH RANDOMLY GENERATED OBSTACLE POSITIONS.

\begin{tabular}{ccccccccc}
\hline Approach & Collision rate & Target reach & Average speed $(\mathrm{m} / \mathrm{s})$ & Training time $(\mathrm{s})$ & Episode & Samples & Training scenario & Deployment scenario \\
\hline CPO & 0.1 & 0.9 & 0.82 & $2.4 \mathrm{e} 4$ & 1000 & $3.3 \mathrm{e} 5$ & Safety Gym & Safety Gym \\
TRPO-L & 0.095 & 0.905 & 0.85 & $2.8 \mathrm{e} 4$ & 1000 & $3.3 \mathrm{e} 5$ & Safety Gym & Safety Gym \\
PPO-L & 0.095 & 0.905 & 0.84 & $2.7 \mathrm{e} 4$ & 1000 & $3.3 \mathrm{e} 5$ & Safety Gym & Safety Gym \\
DDPG-CS & - & - & - & $2.8 \mathrm{e} 4$ & 1000 & $3.3 \mathrm{e} 5$ & with data from (37) & - \\
SAC-CS & - & - & - & $2.8 \mathrm{e} 4$ & 1000 & $3.3 \mathrm{e} 5$ & with data from $(37)$ & - \\
Ours without MPE & $\mathbf{0 . 0 2 5}$ & $\mathbf{0 . 9 7 5}$ & 0.76 & $\mathbf{1 . 7}$ & $\mathbf{1 0 0}$ & $\mathbf{5 e 3}$ & with model $(37)$ & Safety Gym \\
Ours & $\mathbf{0 . 0 3}$ & $\mathbf{0 . 9 7}$ & 0.8 & - & - & - & - & - \\
\hline
\end{tabular}

TABLE II

NUMERICAL COMPARISONS IN SAFETY GYM WITH GENERATED OBSTACLES ON THE PATH BETWEEN THE TARGET AND VEHICLE.

\begin{tabular}{ccccccc}
\hline Approach & Collision rate & Target reach & Average speed $(\mathrm{m} / \mathrm{s})$ & Training time $(\mathrm{s})$ & Episode & Samples \\
\hline CPO & 0.815 & 0.185 & 0.76 & $2.4 \mathrm{e} 4$ & 1000 & $3.3 \mathrm{e} 5$ \\
TRPO-L & 0.835 & 0.165 & 0.8 & $2.8 \mathrm{e} 4$ & 1000 & $3.3 \mathrm{e} 5$ \\
PPO-L & 0.8 & 0.2 & 0.78 & $2.7 \mathrm{e} 4$ & 1000 & $3.3 \mathrm{e} 5$ \\
Ours without MPE & $\mathbf{0 . 2 3}$ & $\mathbf{0 . 7 7}$ & 0.76 & $\mathbf{1 . 7}$ & $\mathbf{1 0 0}$ & $\mathbf{5 e 3}$ \\
Ours & $\mathbf{0}$ & $\mathbf{0 . 5 9 5}$ & 0.76 & - & - & - \\
\hline
\end{tabular}
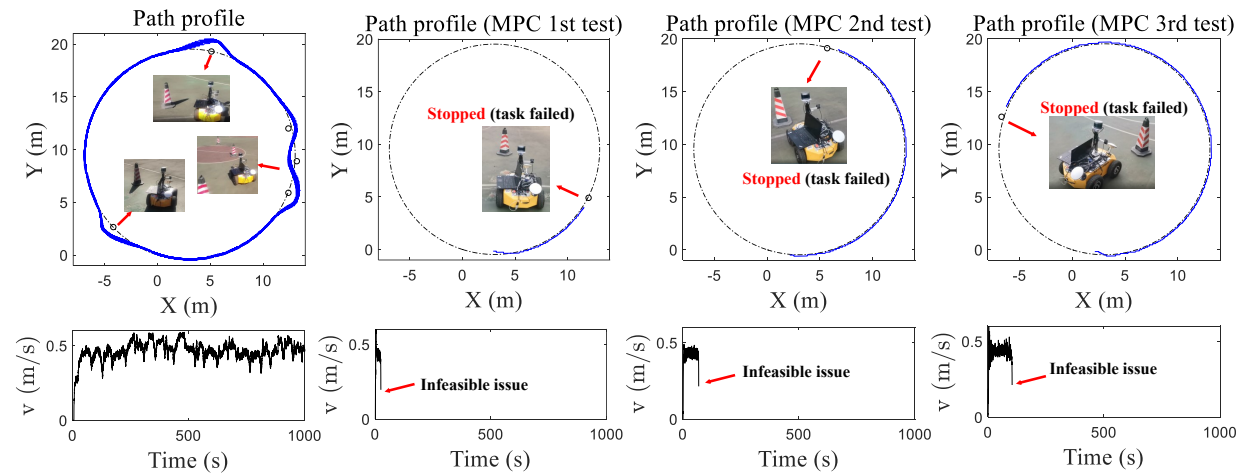

Fig. 2. Path following of the differential-drive vehicle with collision avoidance in the experimental tests. The black dot-dashed line is the circular reference path; the blue lines are the real paths of the vehicle; the small circles are the expanded obstacles (with the vehicle width); the pictures are the screenshots in the collision avoidance process. Our approach successfully run 5 rounds while MPC failed in the first collision avoidance process.

directly deployed the offline learned control policy using the kinematics model in implementation since we did not know the vehicle's dynamic model. All the comparative algorithms were trained and deployed using the same environment in Safety Gym. The simulation results in Table I show that our approach outperforms all the comparative algorithms in data-efficiency, collision avoidance, and performance, see the video details ${ }^{2}$.

Remark 7: There are failures under our offline learned policy without MPE for following reasons. First, the obstacles' locations were generated randomly without considering the vehicle's physical limit. Second, the estimated uncertainty is inaccurate since the inputs in Safety Gym are saturated values with unknown physical interpretations. These issues lead to Theorem 2 being not verified. In our case, one can guarantee safety (possibly with conservativeness) by activating our MPE mechanism even if the model is inaccurate, see Table I and II.

As shown in Table II, when the obstacles were overlapped with the reference path between the target and vehicle, our approach offers a significant performance improvement compared with other adopted approaches ${ }^{2}$. The DDPG-CS and SAC-CS were failed to obtain the converged policy after

\footnotetext{
${ }^{2}$ https://youtube.com/playlist?list=PLPE5-2sIdTlhk5r0VQr66PBEqpAXvdAx. or https://pan.baidu.com/s/1NxJ-zgD4ZdVvqIXQgJkCqg, with extracting code: 9426.
}

several training trials. Therefore we are unable to show the results in the Tables.

Real-world experimental results. We also tested our proposed algorithm on a real-world differential-drive vehicle platform. The control task is to follow a predefined circular path and avoid collisions. In the experiment, we directly deployed the offline learning policy to control the vehicle. The vehicle was equipped with a Laptop in which the Ubuntu operating system was installed. The sampling interval was set as $\Delta t=0.1$ s. At each sampling instant, the onboard laptop computed the control input in real-time using the state information, which was periodically measured by the onboard satellite inertial guidance integrated positioning system (SIGIPS). To simplify the experimental setup, we directly use the SIGIPS to pre-detect the locations of the five obstacles. Additive uniformly distributed measurement noises in the interval $[-0.75 \mathrm{~m}, 0.75 \mathrm{~m}]$ were added.

Also, a standard MPC algorithm was adopted for comparison. Similar to our approach, the cost in MPC is also reformulated with barrier functions for representing the nonconvex obstacle constraint, resulting in an MPC problem like that in [45]. The MPC problem was solved at each sampling interval with an Ipopt solver. Experimental results under collision avoidance were illustrated in Fig. 2, which shows that 

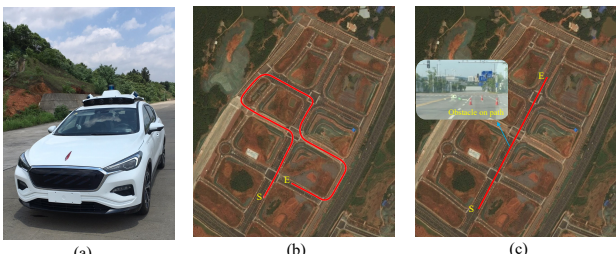

Fig. 3. (a): The HongQi EHS3 autonomous driving experimental platform; (b) the road map with road boundary constraints, where $S$ stands for the starting point, $\mathrm{E}$ stands for the ending point, and the red line is the reference path for path following control; (c) the road map with collision avoidance scenario.

the vehicle with our approach can successfully avoid all obstacles under measurement noises ${ }^{2}$. However, the comparative MPC algorithm stopped in front of the encountered obstacle, leading to an infeasible issue. In other words, MPC failed in completing the path following task ${ }^{2}$ due to the nonconvex obstacle constraint. Moreover, in the unconstrained control case, the root-mean-square cost $J_{e}=\sqrt{1 / M \sum_{j=1}^{M}\|e\|^{2}}$ with $M=800$ of our approach was 0.08 , much smaller than that $\left(J_{e}=0.29\right)$ in MPC, which reveals that our approach performed much better than MPC. This is probably due to the online computational delay of MPC under nonlinear and nonconvex model constraints.

\section{B. Application of an Ackermann-drive vehicle with dynamics model: online learning case}

Consider the path following control of an Ackermanndrive vehicle with collision avoidance. Its simplified lateral dynamics is described by a "bicycle" model (cf. [60]), that is

$$
\begin{aligned}
\dot{X} & =v_{x} \cos \varphi-v_{y} \sin \varphi \\
\dot{Y} & =v_{x} \sin \varphi+v_{y} \cos \varphi \\
\dot{v}_{y} & =-v_{x} \dot{\varphi}+\frac{2}{m}\left[C_{f}\left(\delta-\frac{v_{y}+l_{f} \dot{\varphi}}{v_{x}}\right)+C_{r} \frac{l_{r} \dot{\varphi}-v_{y}}{v_{x}}\right] \\
\ddot{\varphi} & =\frac{2}{I_{z}}\left[l_{f} C_{f}\left(\delta-\frac{v_{y}+l_{f} \dot{\varphi}}{v_{x}}\right)-l_{r} C_{r} \frac{l_{r} \dot{\varphi}-v_{y}}{v_{x}}\right]
\end{aligned}
$$

where $X$ and $Y$ are the coordinates of vehicle center of mass in the Cartesian frame $X o Y, v_{x}$ and $v_{y}$ are the longitudinal and lateral velocities respectively, $\varphi$ is the yaw angle, $I_{z}=$ $4175 \mathrm{~kg} \cdot \mathrm{m}^{2}$ is the yaw moment of inertia, $m=1723 \mathrm{~kg}$ is the mass of vehicle, $C_{f}=66900 N$ and $C_{r}=62700 N$ are the cornering stiffness of the front and rear tires, respectively, $l_{f}=1.322 \mathrm{~m}, l_{r}=1.468 \mathrm{~m}, \delta$ is the front wheel angle variable to be manipulated.

Given path reference points $\left(X^{r}, Y^{r}\right)$ and $v_{x}$, we aim to minimize the lateral distance from the vehicle center of mass to the nearest reference point while avoiding potential collisions with obstacles. To this end, let the nearest point be $\left(X_{p}^{r}, Y_{p}^{r}\right)$, then one can compute the reference yaw angle $\varphi_{p}^{r}$. Define $e_{y}=-\left(X-X_{p}^{r}\right) \sin \left(\varphi_{p}^{r}\right)+\left(Y-Y_{p}^{r}\right) \cos \left(\varphi_{p}^{r}\right), e_{\varphi}=\varphi-\varphi_{p}^{r}$. Let $x=\left(e_{y}, \dot{e}_{y}, e_{\varphi}, \dot{e}_{\varphi}\right)$, then one can obtain the continuoustime lateral dynamical model:

$$
\dot{x}=F_{1}(x)+F_{2}(x) \delta+F_{3}(x) \varphi_{p}^{r}
$$

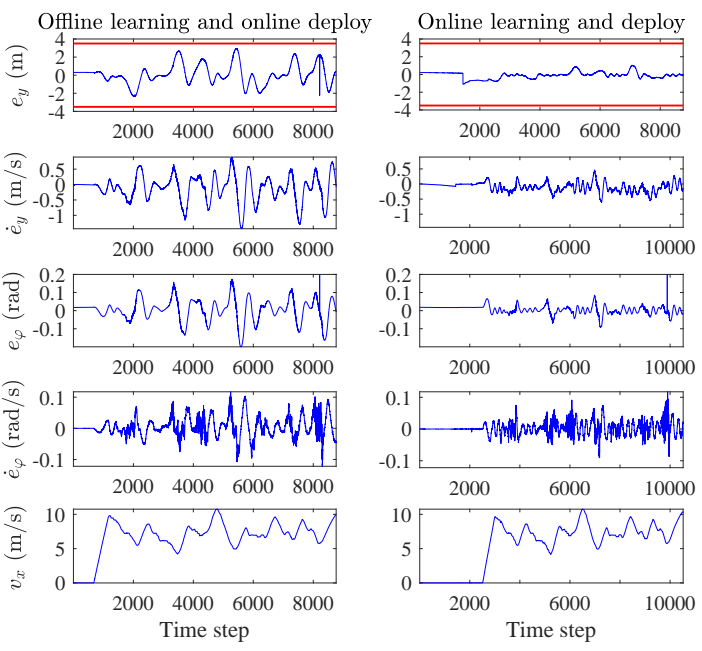

Fig. 4. Variations of the state variables and longitudinal speed of path following with road boundaries (red line) in the experimental tests. Compared with offline learning case, a significantly improved performance can be achieved by online policy learning.
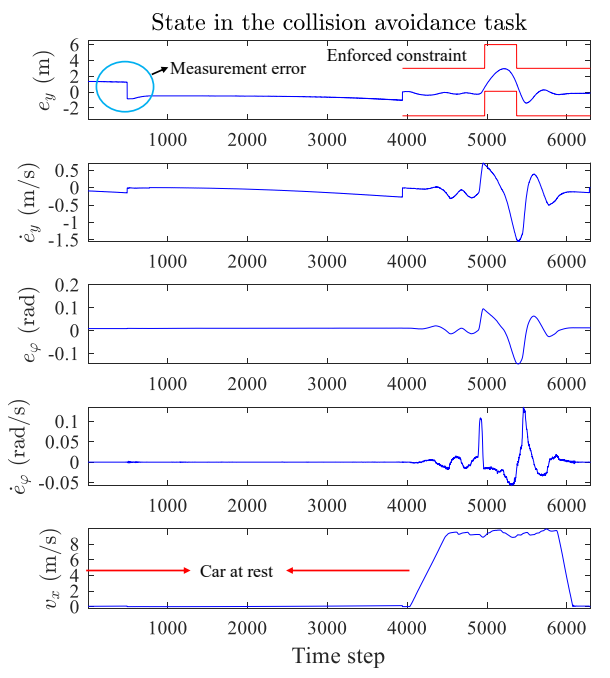

Fig. 5. Experimental results of the integrated path following and collision avoidance: variation of the state variables and longitudinal speed.

where $F_{1}(0)=0, F_{3}(0) \neq 0$. Since $(x, \delta)=0$ might not be an equilibrium point if $\varphi_{p}^{r} \neq 0$, we introduced a virtual control variable $u=\delta+\delta_{f}$, where $\delta_{f}$ was selected such that $F_{2}(x) \delta_{f}=F_{3}(x) \varphi_{p}^{r}$. Consequently, (39) was discretized with a sampling interval $\Delta t=0.02 s$, i.e., $x_{k+1}=x_{k}+\Delta t F_{1}\left(x_{k}\right)+\Delta t F_{2}\left(x_{k}\right) u_{k}$. In the path following control task with collision avoidance, the cost function was chosen as $\bar{J}=\sum_{k=0}^{+\infty}\left\|x_{k}\right\|_{Q}^{2}+\left\|u_{k}\right\|_{R}^{2}+\mu \mathcal{B}_{k}\left(e_{y, k}\right)$, where $Q=I, R=1, \mu=0.02$.

Real-world experimental results ${ }^{2}$. We also tested our safe $\mathrm{RL}$ algorithm on the real-world intelligent vehicle platform built with a HongQi EHS3 electric car to realize the path following control, see Fig. 3. In the experiment, the states of the vehicle were measured by a SIGIPS; then, the measured states were transmitted to an industrial control computer, where the control policy was computed using our approach 
with a sampling interval of $0.02 \mathrm{~s}$. We first applied our algorithm to follow a reference path with road boundaries (see Fig. 3). Different from that in the simulation tests, the vehicle speed was controlled by a PI controller to track a timevarying speed reference. This caused a strong nonlinearity of the lateral dynamics, leading to extra difficulties of the control task. The experimental results displayed in Fig. 4 show that the control policy of our approach can be learned offline and deployed online safely, showing an impressive sim-to-real transfer capability. It is shown in Fig. 4 that one can achieve better control performance by online learning the control policy, which further demonstrates the adaptability of our approach to dynamic environments.

To show the capability of dealing with time-varying state constraints, we tested our approach to track a reference path overlapped with obstacles, see Fig. 3. (c). Similarly, the location information of obstacles was assumed to be predetected. In the experiment, the control policy was learned and deployed synchronously online. The initial constraints were the road boundaries. Then, the constraint on $e_{y}$ was changed accordingly once the vehicle was near the obstacle. Experimental results in Fig. 5 show that the vehicle using our approach can avoid collision successfully and converge rapidly to the reference path after completing the collision avoidance task. Also, as shown in Fig. 5, there were significant measurement errors of the positioning measurement system even though the vehicle was in static and at rest status. Even so, our proposed algorithm showed satisfactory robustness performance under positioning measurement errors.

\section{Implementation issues and discussions}

Implementation issues. First, the tuning parameter $\mu$ is suggested to be chosen smaller than the entries of $Q$ and $R$ to obtain a satisfactory control performance. A larger choice of $\mu$ might result in a safe but conservative control policy. Second, the initial values of $W_{a, \sigma}, \hat{K}$, and $\hat{\rho}$ in the actor must be properly selected such that the initial control policy with (16) is $L$-step safe, which is a prior condition in Theorem 1. Finally, the relaxing factor $\kappa_{b}$ in Lemma 1 must also be selected properly. A smaller choice is suggested if a less conservative control policy is expected, while a larger choice can be made to ensure absolute control safety.

Discussions. Note that a predefined model is used for learning a control policy with our approach. The model can be a physics-based model that is used in this work or derived using data-driven techniques [40]. In the latter case, no prior dynamics information is required, leading to an indirect datadriven controller design. Note that, as another prominent feature, our approach can learn an explicit control policy offline and deploy it to a different control scenario even if the concerned constraints are nonlinear and nonconvex. However, in MPC, the control action must be computed online by periodically solving an optimization problem [40], which can be difficult for the on-the-fly implementation under nonlinear and nonconvex constraints, see Section V-A. As shown in the simulation and real-world experiments, our learned policy using an inaccurate model shows an impressive sim-to-real transfer capability compared with state-of-the-art model-free RL approaches. Indeed, our approach is a step forward in applying safe RL to the real-world intelligent vehicle control problem.

\section{CONCLUSIONS}

This paper proposed a safe RL algorithm with a barrierbased control policy structure and a multi-step policy evaluation mechanism for the optimal control of discrete-time nonlinear systems with time-varying safety constraints. Under certain conditions, safety can be guaranteed by our approach in both online and offline learning cases. Our approach can solve continuous control tasks in the environment with abruptly changes, both online and offline. The convergence and robustness of our safe RL under nominal and disturbed scenarios were proven, respectively. The convergence condition of the barrier-based actor-critic learning algorithm was obtained.

Besides numerical simulations for theoretical verification, we tested our approach in two real-world intelligent vehicle platforms. The simulation and real-world experiment results illustrate that our method outperforms state-of-the-art safe RL approaches in control safety, shows an impressive simto-real transfer capability and a satisfactory real-world online learning performance. In general, the proposed safe RL is a step forward in applying safe RL to the optimal control of real-world nonlinear physical systems with time-varying safety constraints. Future works will consider the extension to modelfree safe RL with theoretical guarantees.

\section{APPENDIX}

\section{A. Simulation on regulation of a mass-point robot}

Consider the regulation control of a mass-point robot. Its discrete-time model is described by $x_{k+1}=A x_{k}+B u_{k}$ where

$$
A=\left[\begin{array}{cc}
0.995 & 0.0998 \\
-0.0998 & 0.995
\end{array}\right], B=\left[\begin{array}{l}
-0.2 \\
-0.1
\end{array}\right],
$$

$x=\left(x_{1}, x_{2}\right)$ are the positions to the references.

TABLE III

COMPARISONS IN TERMS OF SAFETY RATE IN MASS-POINT ROBOT EXAMPLE.

\begin{tabular}{ccc}
\hline Approach & Ours & Safety-aware RL [29] \\
\hline Time-invariant constraint & $100 \%$ & $100 \%$ \\
Time-varying constraint & $100 \%$ & $\leq 95 \%$ \\
\hline
\end{tabular}

In the simulation process, the penalty matrices were selected as $Q=I, R=0.1, \mu=0.001$. The discount factor $\gamma$ was $\gamma=0.95$. The step $L$ was chosen as $L=10$. The entries of weighting matrices $W_{c}$ and $W_{a}$ were initialized with uniformly random numbers. The initial state was $x=(-0.5,-0.5)$. The state and control were initially limited by $-1 \leq x_{1}, x_{2} \leq$ $0.5, \quad-1 \leq u \leq 0.3$. Then at $k=285, x$ was reset as $x=$ $(-0.65,-0.65)$ and the constraints were changed as $-0.5 \leq$ $x_{1}, x_{2} \leq 0.3,-0.5 \leq u \leq 0.1$.

The control performances are compared in Fig. 6, which shows that all approaches can converge to the origin. In contrast, our approach and safety-aware RL [29] can ensure 

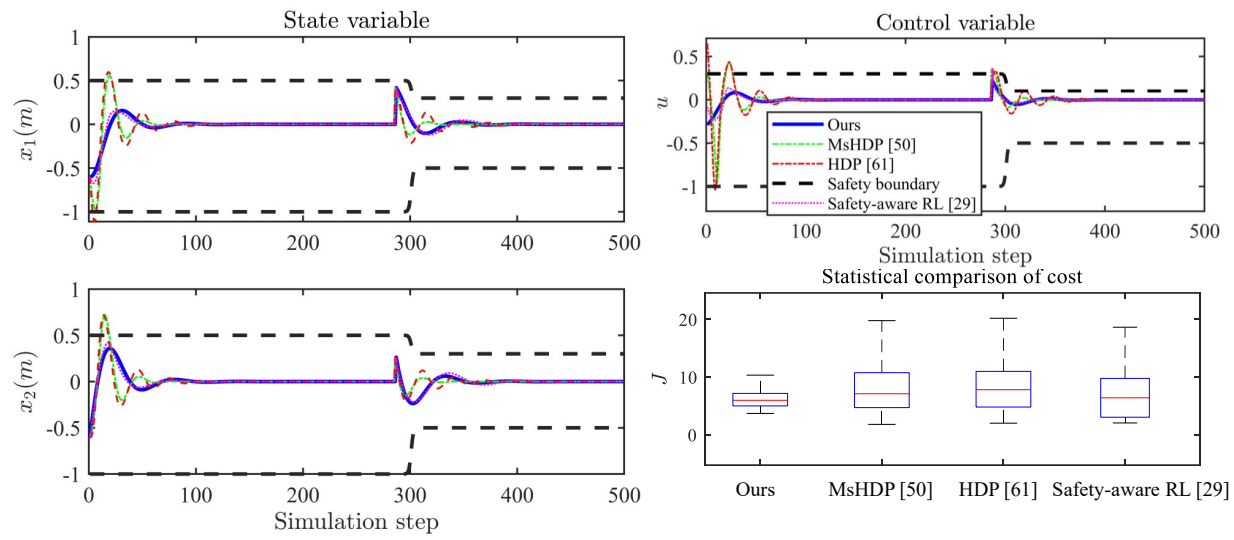

Fig. 6. Simulation results for control of mass-point robot: The state, control, and cost comparisons between our approach and algorithms in [29], [50], and [61]. Right-bottom panel: Statistical comparison of cost with 500 repetitive experiments; red lines stand for mean value of $J$, black boxes are the area of standard deviations of $J$, while black horizontal lines are the maximal and minimal deviations.

safety constraint satisfaction in the control process. Moreover, comparisons in terms of safety for 500 repetitive experimental tests are listed in Table III, which illustrates that our approach can ensure safety for all the performed tests, while it did not hold so for safety-aware RL in [29] under time-varying safety constraints. The reason behind this is the adopted multistep policy evaluation mechanism in our approach. From the box-plot in the right-bottom panel of Fig. 6, one can see that the mean values of $J$ in all approaches are comparable and its standard cost deviation in our approach is smaller than that in [29], [50], and [61]. The results reveal that the performance of our approach is more stable under state and control constraints due to the proposed barrier-based control policy design.

\section{B. Simulation on regulation of Van der Pol oscillator}

Consider the regulation control of Van der Pol oscillator [40]. Its discrete-time model is given as

$$
\left\{\begin{array}{l}
x_{1, k+1}=x_{1, k}+\Delta t x_{2, k} \\
x_{2, k+1}=x_{2, k}+\Delta t\left(x_{2, k}-x_{1, k}^{2} x_{2, k}-x_{1, k}+u_{k}\right)
\end{array}\right.
$$

where $x_{1}$ and $x_{2}$ are the states, and $u$ is the control variable, $\Delta t=0.01 \mathrm{~s}$. In the simulation process, the penalty matrices were selected as $Q=I, R=0.1, \mu=0.01$. The discount factor $\gamma$ was $\gamma=0.95$. The step $L$ was chosen as $L=10$. The entries of weighting matrices $W_{c}$ and $W_{a}$ were initialized using saturated uniformly random numbers such that Theorem 1 was fulfilled. Starting with an initial condition $x_{0}=(-0.5,-0.5)$, the training was performed under timevarying state constraints, see Fig. 7.

TABLE IV

COMPARISONS IN TERMS OF SAFETY RATE IN VAN DER POL OSCILLATOR.

\begin{tabular}{ccc}
\hline Approach & Ours & Safety-aware RL [29] \\
\hline $\begin{array}{c}\text { Time-invariant constraint } \\
\text { Time-varying constraint }\end{array}$ & $100 \%$ & $100 \%$ \\
& $100 \%$ & $\leq 50 \%$ \\
\hline
\end{tabular}

We compared our approach with two classic control methods, i.e., heuristic dynamic programming (HDP) [61], multistep heuristic dynamic programming (MsHDP) [50] and three

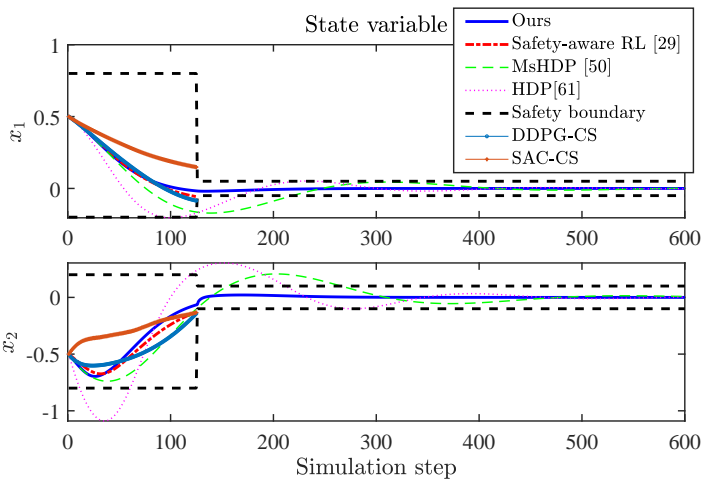

Fig. 7. Simulation results for control of Van der Pol oscillator: The state variables (left panel) and the stage cost (right panel) with our approach and adopted comparative algorithms. In each training of safety-aware RL [29], DDPG-CS, and SAC-CS, the control (learning) safety was not fulfilled, and the learning process was terminated when the size of state constraint was suddenly reduced. However, our approach can adapt to the constraint variation due to the adopted control policy structure and multi-step policy evaluation mechanism.

safe RL approaches, e.g., safety-aware RL [29], DDPG-CS and SAC-CS. The control parameters of the proposed safe RL and the comparative approaches in [29], [50], and [61] were set similar. In DDPG-CS and SAC-CS, the cost function was reshaped with the same barrier functions adopted in the paper, and all the training parameters are fine-tuned according to [59] and [3] respectively. The simulation results in Fig. 7 and Table IV show that our approach can cope with timevarying state constraints in the control (learning) process while safety-aware RL in [29], DDPG-CS, and SAC-CS could fail due to the sudden change of constraints. The reason behind this is that the adopted safe RL approaches could not predict future changes of safety constraints and inform how to achieve safety by the actor-critic structure, hence prone to failing in abruptly changed environments. MsHDP in [50] and HDP in [61] can not guarantee safety constraint satisfaction. Moreover, our approach converged faster than that in [29], [50], and [61], see Fig. 7. 


\section{REFERENCES}

[1] Ivo Grondman, Lucian Busoniu, Gabriel AD Lopes, and Robert Babuska. A survey of actor-critic reinforcement learning: Standard and natural policy gradients. IEEE Transactions on Systems, Man, and Cybernetics, Part C (Applications and Reviews), 42(6):1291-1307, 2012.

[2] Sangwoon Kim, David Donghyun Kim, and Brian Anthony. Dynamic control of a fiber manufacturing process using deep reinforcement learning. IEEE/ASME Transactions on Mechatronics, 2021.

[3] Tuomas Haarnoja, Aurick Zhou, Pieter Abbeel, and Sergey Levine. Soft actor-critic: Off-policy maximum entropy deep reinforcement learning with a stochastic actor. In International conference on machine learning, pages 1861-1870. PMLR, 2018.

[4] Derong Liu and Qinglai Wei. Policy iteration adaptive dynamic programming algorithm for discrete-time nonlinear systems. IEEE Transactions on Neural Networks and Learning Systems, 25(3):621-634, 2013.

[5] Huaguang Zhang, Yanhong Luo, and Derong Liu. Neural-network-based near-optimal control for a class of discrete-time affine nonlinear systems with control constraints. IEEE Transactions on Neural Networks, 20(9):1490-1503, 2009.

[6] Yu Jiang and Zhong-Ping Jiang. Computational adaptive optimal control for continuous-time linear systems with completely unknown dynamics. Automatica, 48(10):2699-2704, 2012.

[7] Jaehyun Lim, Seungchul Ha, and Jongeun Choi. Prediction of reward functions for deep reinforcement learning via gaussian process regression. IEEE/ASME Transactions on Mechatronics, 25(4):1739-1746, 2020.

[8] Hui Wang, Jiawen Xu, Chuan Sun, Ruqiang Yan, and Xuefeng Chen. Intelligent fault diagnosis for planetary gearbox using time-frequency representation and deep reinforcement learning. IEEE/ASME Transactions on Mechatronics, 2021.

[9] Javier Garcia and Fernando Fernández. A comprehensive survey on safe reinforcement learning. Journal of Machine Learning Research, 16(1):1437-1480, 2015.

[10] Yinlam Chow, Ofir Nachum, Aleksandra Faust, Edgar Duenez-Guzman, and Mohammad Ghavamzadeh. Lyapunov-based safe policy optimization for continuous control. arXiv preprint arXiv:1901.10031, 2019.

[11] Yinlam Chow, Ofir Nachum, Edgar Duenez-Guzman, and Mohammad Ghavamzadeh. A Lyapunov-based approach to safe reinforcement learning. arXiv preprint arXiv:1805.07708, 2018.

[12] Felix Berkenkamp, Matteo Turchetta, Angela P Schoellig, and Andreas Krause. Safe model-based reinforcement learning with stability guarantees. Advances in Neural Information Processing Systems 30, 2:909-919, 2018.

[13] Krishnan Srinivasan, Benjamin Eysenbach, Sehoon Ha, Jie Tan, and Chelsea Finn. Learning to be safe: Deep RL with a safety critic, 2020.

[14] Ming Yu, Zhuoran Yang, Mladen Kolar, and Zhaoran Wang. Convergent policy optimization for safe reinforcement learning. arXiv preprint arXiv:1910.12156, 2019.

[15] Tengyu Xu, Yingbin Liang, and Guanghui Lan. Crpo: A new approach for safe reinforcement learning with convergence guarantee. In International Conference on Machine Learning, pages 11480-11491. PMLR, 2021.

[16] Haitong Ma, Jianyu Chen, Shengbo Eben Li, Ziyu Lin, Yang Guan, Yangang Ren, and Sifa Zheng. Model-based constrained reinforcement learning using generalized control barrier function. arXiv preprint arXiv:2103.01556, 2021.

[17] Thiago D Simão, Nils Jansen, and Matthijs TJ Spaan. Alwayssafe: Reinforcement learning without safety constraint violations during training. 2021.

[18] Sanae Amani, Christos Thrampoulidis, and Lin F Yang. Safe reinforcement learning with linear function approximation. arXiv preprint arXiv:2106.06239, 2021.

[19] Tsung-Yen Yang, Justinian Rosca, Karthik Narasimhan, and Peter J Ramadge. Accelerating safe reinforcement learning with constraintmismatched baseline policies. In International Conference on Machine Learning, pages 11795-11807. PMLR, 2021.

[20] Subin Huh and Insoon Yang. Safe reinforcement learning for probabilistic reachability and safety specifications: A Lyapunov-based approach. arXiv preprint arXiv:2002.10126, 2020.

[21] Liyuan Zheng, Yuanyuan Shi, Lillian J Ratliff, and Baosen Zhang. Safe reinforcement learning of control-affine systems with vertex networks. In Learning for Dynamics and Control, pages 336-347. PMLR, 2021.

[22] Zahra Marvi and Bahare Kiumarsi. Safe reinforcement learning: A control barrier function optimization approach. International Journal of Robust and Nonlinear Control, 31(6):1923-1940, 2021.
[23] Baiming Chen, Zuxin Liu, Jiacheng Zhu, Mengdi Xu, Wenhao Ding, Liang $\mathrm{Li}$, and Ding Zhao. Context-aware safe reinforcement learning for non-stationary environments. In 2021 IEEE International Conference on Robotics and Automation (ICRA), pages 10689-10695. IEEE, 2021.

[24] Yutong Li, Nan Li, H Eric Tseng, Anouck Girard, Dimitar Filev, and Ilya Kolmanovsky. Safe reinforcement learning using robust action governor. In Learning for Dynamics and Control, pages 1093-1104. PMLR, 2021.

[25] Lukas Brunke, Melissa Greeff, Adam W Hall, Zhaocong Yuan, Siqi Zhou, Jacopo Panerati, and Angela P Schoellig. Safe learning in robotics: From learning-based control to safe reinforcement learning. arXiv preprint arXiv:2108.06266, 2021.

[26] Spencer M Richards, Felix Berkenkamp, and Andreas Krause. The Lyapunov neural network: Adaptive stability certification for safe learning of dynamical systems. In Conference on Robot Learning, pages 466-476. PMLR, 2018.

[27] Changyin Sun, Wei He, and Jie Hong. Neural network control of a flexible robotic manipulator using the lumped spring-mass model. IEEE Transactions on Systems, Man, and Cybernetics: Systems, 47(8):18631874, 2016.

[28] Linghuan Kong, Wei He, Chenguang Yang, Zhijun Li, and Changyin Sun. Adaptive fuzzy control for coordinated multiple robots with constraint using impedance learning. IEEE transactions on cybernetics, 49(8):3052-3063, 2019.

[29] Yongliang Yang, Yixin Yin, Wei He, Kyriakos G Vamvoudakis, Hamidreza Modares, and Donald C Wunsch. Safety-aware reinforcement learning framework with an actor-critic-barrier structure. In 2019 American Control Conference (ACC), pages 2352-2358. IEEE, 2019.

[30] Nathan Fulton and André Platzer. Safe reinforcement learning via formal methods: Toward safe control through proof and learning. In Proceedings of the AAAI Conference on Artificial Intelligence, volume 32, 2018.

[31] Matteo Turchetta, Andrey Kolobov, Shital Shah, Andreas Krause, and Alekh Agarwal. Safe reinforcement learning via curriculum induction. arXiv preprint arXiv:2006.12136, 2020.

[32] Mohammed Alshiekh, Roderick Bloem, Rüdiger Ehlers, Bettina Könighofer, Scott Niekum, and Ufuk Topcu. Safe reinforcement learning via shielding. In Proceedings of the AAAI Conference on Artificial Intelligence, volume 32, 2018.

[33] Mario Zanon and Sébastien Gros. Safe reinforcement learning using robust MPC. IEEE Transactions on Automatic Control, 2020.

[34] Brijen Thananjeyan, Ashwin Balakrishna, Suraj Nair, Michael Luo, Krishnan Srinivasan, Minho Hwang, Joseph E Gonzalez, Julian Ibarz, Chelsea Finn, and Ken Goldberg. Recovery RL: Safe reinforcement learning with learned recovery zones. IEEE Robotics and Automation Letters, 6(3):4915-4922, 2021.

[35] William Saunders, Girish Sastry, Andreas Stuhlmueller, and Owain Evans. Trial without error: Towards safe reinforcement learning via human intervention, 2017.

[36] Nolan Wagener, Byron Boots, and Ching-An Cheng. Safe reinforcement learning using advantage-based intervention. arXiv preprint arXiv:2106.09110, 2021.

[37] Yichen Chen and Mengdi Wang. Stochastic primal-dual methods and sample complexity of reinforcement learning. arXiv preprint arXiv:1612.02516, 2016.

[38] Santiago Paternain, Miguel Calvo-Fullana, Luiz FO Chamon, and Alejandro Ribeiro. Safe policies for reinforcement learning via primal-dual methods. arXiv preprint arXiv:1911.09101, 2019.

[39] Dongsheng Ding, Xiaohan Wei, Zhuoran Yang, Zhaoran Wang, and Mihailo Jovanovic. Provably efficient safe exploration via primaldual policy optimization. In International Conference on Artificial Intelligence and Statistics, pages 3304-3312. PMLR, 2021.

[40] Xinglong Zhang, Wei Pan, Riccardo Scattolini, Shuyou Yu, and Xin Xu. Robust tube-based model predictive control with koopman operatorsextended version. arXiv preprint arXiv:2108.13011 (Accepted for publication at Automatica), 2021.

[41] Peter Geibel and Fritz Wysotzki. Risk-sensitive reinforcement learning applied to control under constraints. Journal of Artificial Intelligence Research, 24:81-108, 2005.

[42] Anand Balakrishnan and Jyotirmoy V Deshmukh. Structured reward shaping using signal temporal logic specifications. In 2019 IEEE/RSJ International Conference on Intelligent Robots and Systems (IROS), pages 3481-3486. IEEE, 2019.

[43] Yujing Hu, Weixun Wang, Hangtian Jia, Yixiang Wang, Yingfeng Chen, Jianye Hao, Feng Wu, and Changjie Fan. Learning to utilize shaping rewards: A new approach of reward shaping. arXiv preprint arXiv:2011.02669, 2020.

[44] Chen Tessler, Daniel J Mankowitz, and Shie Mannor. Reward constrained policy optimization. arXiv preprint arXiv:1805.11074, 2018. 
[45] Adrian G Wills and William P Heath. Barrier function based model predictive control. Automatica, 40(8):1415-1422, 2004.

[46] Qisong Yang, Thiago D Simão, Simon H Tindemans, and Matthijs TJ Spaan. Wcsac: Worst-case soft actor critic for safety-constrained reinforcement learning. In Proceedings of the Thirty-Fifth AAAI Conference on Artificial Intelligence. AAAI Press, online, 2021.

[47] Yongliang Yang, Da-Wei Ding, Haoyi Xiong, Yixin Yin, and Donald C Wunsch. Online barrier-actor-critic learning for H-infinity control with full-state constraints and input saturation. Journal of the Franklin Institute, 357(6):3316-3344, 2020.

[48] Huaguang Zhang, Ruizhuo Song, Qinglai Wei, and Tieyan Zhang. Optimal tracking control for a class of nonlinear discrete-time systems with time delays based on heuristic dynamic programming. IEEE Transactions on Neural Networks, 22(12):1851-1862, 2011.

[49] Xiangnan Zhong, Zhen Ni, and Haibo He. A theoretical foundation of goal representation heuristic dynamic programming. IEEE Transaction on Neural Networks and Learning Systems, 27(12):2513-2525, 2015.

[50] Biao Luo, Derong Liu, Tingwen Huang, and Jiangiiang Liu. Output tracking control based on adaptive dynamic programming with multistep policy evaluation. IEEE Transactions on Systems, Man, and Cybernetics: Systems, 49(10):2155-2165, 2017.

[51] Alexander I Cowen-Rivers, Daniel Palenicek, Vincent Moens, Mohammed Abdullah, Aivar Sootla, Jun Wang, and Haitham Ammar Samba: Safe model-based \& active reinforcement learning. arXiv preprint arXiv:2006.09436, 2020.

[52] Syed Ali Asad Rizvi and Zongli Lin. Output feedback Q-learning control for the discrete-time linear quadratic regulator problem. IEEE Transactions on Neural Networks and Learning Systems, 30(5):15231536, 2018

[53] D Limon Marruedo, T Alamo, and EF Camacho. Input-to-state stable MPC for constrained discrete-time nonlinear systems with bounded additive uncertainties. In Proceedings of the 41st IEEE Conference on Decision and Control, 2002., volume 4, pages 4619-4624. IEEE, 2002.

[54] Antonio Sgorbissa. Integrated robot planning, path following, and obstacle avoidance in two and three dimensions: Wheeled robots, underwater vehicles, and multicopters. The International Journal of Robotics Research, 38(7):853-876, 2019.

[55] Lionel Lapierre, Rene Zapata, and Pascal Lepinay. Combined pathfollowing and obstacle avoidance control of a wheeled robot. The International Journal of Robotics Research, 26(4):361-375, 2007.

[56] Alex Ray, Joshua Achiam, and Dario Amodei. Benchmarking safe exploration in deep reinforcement learning. arXiv preprint arXiv:1910.01708, 7, 2019.

[57] Emanuel Todorov, Tom Erez, and Yuval Tassa. Mujoco: A physics engine for model-based control. In 2012 IEEE/RSJ International Conference on Intelligent Robots and Systems, pages 5026-5033. IEEE, 2012.

[58] Joshua Achiam, David Held, Aviv Tamar, and Pieter Abbeel. Constrained policy optimization. In International Conference on Machine Learning, pages 22-31. PMLR, 2017.

[59] Timothy P Lillicrap, Jonathan J Hunt, Alexander Pritzel, Nicolas Heess, Tom Erez, Yuval Tassa, David Silver, and Daan Wierstra Continuous control with deep reinforcement learning. arXiv preprint arXiv:1509.02971, 2015.

[60] Rajesh Rajamani. Vehicle dynamics and control. Springer Science \& Business Media, 2011.

[61] Huaguang Zhang, Qinglai Wei, and Yanhong Luo. A novel infinite-time optimal tracking control scheme for a class of discrete-time nonlinear systems via the greedy HDP iteration algorithm. IEEE Transactions on Systems, Man, and Cybernetics, Part B (Cybernetics), 38(4):937-942, 2008.

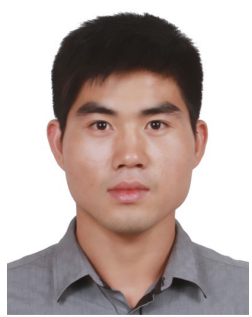

Xinglong Zhang (M'19) received the Ph.D. degree in System and Control from Politecnico di Milano, Italy, 2018. He is presently an Associate Professor at the College of Intelligence Science and Technology, National University of Defense Technology, Changsha, China. His research interests include safe reinforcement learning, model predictive control, and their applications in automotive systems.

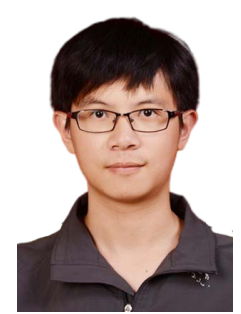

Yaoqian Peng received the B.S. degree in Control engineering from Central South University, Changsha, China, in 2020. He is now pursuing his master's degree in Control Engineering from the National University of Defense Technology, Changsha, China. His research interests include safe reinforcement learning and its applications in robotics.

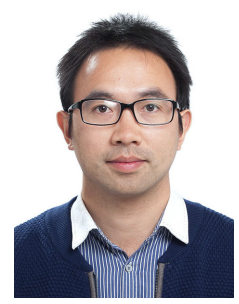

Biao Luo (M'15-SM'18) received the Ph.D. degree in control science and engineering from Beihang University, Beijing, China, in 2014. He is currently a Professor with the School of Automation, Central South University (CSU), Changsha, China. His current research interests include distributed parameter systems, intelligent control, reinforcement learning, deep learning, and computational intelligence. Dr. Luo was a recipient of the Chinese Association of Automation Outstanding Ph.D Dissertation Award in 2015. He serves as an Associate Editor for the IEEE Transactions on Neural Networks and Learning Systems, the IEEE Transactions on Emerging Topics in Computational Intelligence, the Artificial Intelligence Review, the Neurocomputing, and the Journal of Industrial and Management Optimization.

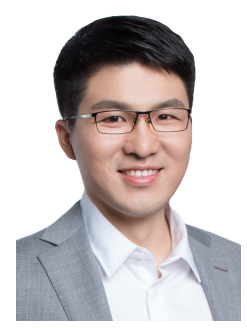

Wei Pan received the Ph.D. degree in Bioengineering from Imperial College London in 2016. He is currently an Assistant Professor at Department of Cognitive Robotics, Delft University of Technology. Until May 2018, he was a Project Leader at DJI, Shenzhen, China, responsible for machine learning research for DJI drones and AI accelerator. He is the recipient of Dorothy Hodgkin's Postgraduate Awards, Microsoft Research Ph.D. Scholarship and Chinese Government Award for Outstanding Students Abroad, Shenzhen Peacock Plan Award. He is on the editorial board of CoRL, ICRA, IROS, IEEE Robotics and Automation Letters. His research interests include machine learning and control theory with applications in robotics.

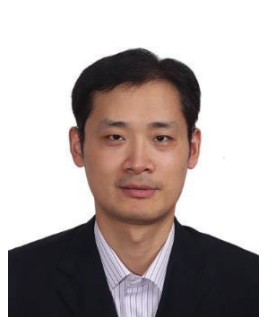

Xin Xu (M'09-SM'12) received the Ph.D. degree in control science and engineering from the College of Mechatronics and Automation, National University of Defense Technology (NUDT), in 2002. He has been a Visiting Professor with Hong Kong Polytechnic University, Hong Kong; the University of Alberta, Edmonton, AB, Canada; the University of Guelph, Guelph, ON, Canada; and the University of Strathclyde, Glasgow, U.K. He is currently a Professor with the College of Intelligence Science and Technology, NUDT. His current research interests include intelligent control, reinforcement learning, approximate dynamic programming, machine learning, robotics, and autonomous vehicles. Dr. Xu was a recipient of the National Science Fund for Outstanding Youth in China and the Second-Class National Natural Science Award of China. He has served as an Associate Editor or a Guest Editor for Information Sciences, International Journal of Robotics and Automation, IEEE Transactions on Systems, Man, and Cybernetics: Systems, Intelligent Automation and Soft Computing, International Journal of Adaptive Control and Signal Processing, and Acta Automatica Sinica. 


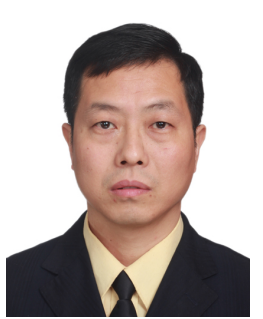

Haibin Xie received the Ph.D. degree in Control Science and Engineering from National University of Defense Technology, Changsha, China, 2006. He is presently an Associate Professor at the College of Intelligence Science and Technology, National University of Defense Technology, Changsha, China. His research interests include machine learning and its applications to automotive systems. 